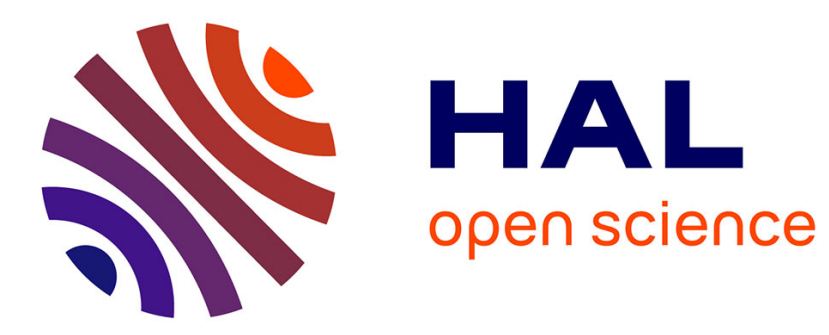

\title{
Uncertainty Quantification for Modal Parameters from Stochastic Subspace Identification on Multi-Setup Measurements
}

\author{
Michael Döhler, Xuan-Binh Lam, Laurent Mevel
}

\section{- To cite this version:}

Michael Döhler, Xuan-Binh Lam, Laurent Mevel. Uncertainty Quantification for Modal Parameters from Stochastic Subspace Identification on Multi-Setup Measurements. Mechanical Systems and Signal Processing, 2013, 36 (2), pp.562-581. 10.1016/j.ymssp.2012.11.011 . hal-00811706

\author{
HAL Id: hal-00811706 \\ https://hal.inria.fr/hal-00811706
}

Submitted on 20 Apr 2020

HAL is a multi-disciplinary open access archive for the deposit and dissemination of scientific research documents, whether they are published or not. The documents may come from teaching and research institutions in France or abroad, or from public or private research centers.
L'archive ouverte pluridisciplinaire HAL, est destinée au dépôt et à la diffusion de documents scientifiques de niveau recherche, publiés ou non, émanant des établissements d'enseignement et de recherche français ou étrangers, des laboratoires publics ou privés. 


\title{
Uncertainty Quantification for Modal Parameters from Stochastic Subspace Identification on Multi-Setup Measurements ${ }^{\text {th }}$
}

\author{
Michael Döhler*, Xuan-Binh Lam, Laurent Mevel \\ Inria, Centre Rennes - Bretagne Atlantique, 35042 Rennes, France
}

\begin{abstract}
In operational modal analysis, the modal parameters (natural frequencies, damping ratios and mode shapes), obtained with stochastic subspace identification from ambient vibration measurements of structures, are subject to statistical uncertainty. It is hence necessary to evaluate the uncertainty bounds of the obtained results, which can be done by a first-order perturbation analysis. To obtain vibration measurements at many coordinates of a structure with only a few sensors, it is common practice to use multiple sensor setups for the measurements. Recently, a multi-setup subspace identification algorithm has been proposed that merges the data from different setups prior to the identification step to obtain one set of global modal parameters, taking the possibly different ambient excitation characteristics between the measurements into account. In this paper, an algorithm is proposed that efficiently estimates the covariances on modal parameters obtained from this multi-setup subspace identification. The new algorithm is validated on multi-setup ambient vibration data of the Z24 Bridge, benchmark of the COST F3 European network.
\end{abstract}

Keywords: System identification, Subspace methods, Modal analysis, Multi-setup measurements, Uncertainty bounds

\section{Introduction}

Stochastic subspace identification methods have been proven efficient for Operational Modal Analysis (OMA), where the modal parameters of mechanical or civil structures (frequencies, damping ratios, mode shapes) are obtained from ambient vibration measurements [1-4]. To obtain vibration measurements at many coordinates of a structure with only a few sensors, it is common practice to use multiple sensor setups for the measurements. For these multisetup measurements, some of the sensors, the so-called reference sensors, stay fixed throughout all the setups, while the other sensors are moved from setup to setup [5-10]. By fusing in some way the corresponding data, this allows to perform modal identification as if there was a very large number of sensors, even in the range of a few hundreds or thousands.

Processing this multi-setup measurement data for structural analysis is often achieved by performing eigenstructure identification for each record separately, and then merging the results obtained for records corresponding to different sensor setups. However, mode matching may be not easy in practice, and like this the result of mode shape gluing may not be consistent [5-7]. An empirical merging approach for covariance-driven subspace identification was described in [8], in which Hankel matrices containing the output correlations of the different setups are combined before doing the global system identification. While this approach has shown good results in several test cases [8-10] and no user interaction to match modes of different setups is necessary, it is lacking some theoretical properties when the ambient excitation properties change between the measurements of the different setups. In [11, 12], a method

\footnotetext{
औ A preliminary version of this paper was presented at the 50th IEEE Conference on Decision and Control and European Control Conference, Orlando, USA, 2011.

${ }^{*}$ Corresponding author. Present address: Northeastern University, Department of Civil and Environmental Engineering, Center for Digital Signal Processing, 360 Huntington Avenue, Boston MA 02115. E-Mail: m.doehler@ neu.edu, Tel. +1 617373 4417, Fax +1 6173734419.

Email addresses: michael .dohler@inria.fr (Michael Döhler), binhlamxuan@yahoo.com (Xuan-Binh Lam), laurent.mevel@inria.fr (Laurent Mevel)
} 
was proposed to merge and to normalize the data from all the setups, before doing the global system identification on it in one step, while taking the possibly different ambient excitation characteristics between the measurements into account. This method was designed for covariance-driven subspace identification and generalized in [13] to a large range of subspace methods, including data-driven subspace methods [2]. With this merging strategy, a theoretically sound and simple identification of the modal parameters is possible. Furthermore, the merging approach can be used in an iterative scheme where no global Hankel or observability matrix is necessary, but the desired system matrices can be computed iteratively setup by setup, which is a computational advantage if the number of setups is large. The method was successfully applied for the modal analysis of large civil structures e.g. in [14-17].

All identified modal parameters are afflicted with statistical uncertainty due to many reasons, such as finite number of data samples, undefined measurement noises, non-stationary excitation, etc. Then the system identification algorithms do not yield the exact system matrices and identification results are subject to variance errors. The identified parameters from subspace identification are asymptotically normal distributed $[18,19]$ and their asymptotic variance was considered in [20]. Covariance estimates on the identified parameters have been obtained in [21-23] based on the propagation of first-order perturbations from the data to the identified parameters. A detailed formulation of the covariance computation for the modal parameters from covariance-driven stochastic subspace identification is given in [23]. The current paper expands on this and focuses on the covariance computation for multi-setup subspace identification with the approach from [13]. While this approach is based on the (theoretical) construction of a global matrix that gathers data from all the setups that can be very large in the case of many setups and sensors, the actual computation of the global matrix can be avoided and the system matrices can be obtained iteratively from the setups. The merging strategy can be written in a modular way, where the matrices of each measurement setup can be processed separately without the need of assembling matrices with data from all setups. As the uncertainty computation depends on the covariance of such matrices, whose dimensions are reduced due to the modular approach but that are still huge as they are quadratic in terms of the underlying matrices, memory problems may arise. In this paper, special attention is paid to the size explosion of the involved covariance matrices for a high number of measurement setups and an efficient uncertainty quantification algorithm is derived, whose computational complexity increases only linearly with the number of setups.

The paper is organized as follows. In Section 2, some preliminary modeling and the general subspace method are stated. In Section 3, the global merging algorithm for measurements from multiple sensor setups is explained. In Section 4, notations and results of the uncertainty computations for a single setup obtained in [23] are recalled. With these foundations, the covariance computation for multi-setup measurements is derived in Section 5. A new normalization scheme for the covariance computation of the mode shapes is proposed in Section 6. In Section 7, the resulting algorithm is applied to the output-only multi-setup system identification of the Z24 Bridge benchmark in order to obtain confidence bounds on the modal parameters.

\section{Stochastic subspace identification (SSI)}

\subsection{Vibration modeling}

The behavior of a vibrating structure is described by a continuous-time, time-invariant, linear dynamical system, modeled by the vector differential system

$$
\left\{\begin{aligned}
\mathcal{M} \ddot{x}(t)+C \dot{x}(t)+\mathcal{K} x(t) & =v(t) \\
y(t) & =L x(t)
\end{aligned}\right.
$$

where $t$ denotes continuous time; $\mathcal{M}, C, \mathcal{K} \in \mathbb{R}^{d \times d}$ are mass, damping, and stiffness matrices, respectively; the (high dimensional) vector $x(t)$ is the displacement vector of the $d$ degrees of freedom of the structure; the external force $v(t)$ is unmeasured; measurements are collected in the (low dimensional) vector $y(t)$ and matrix $L \in \mathbb{R}^{r \times d}$ indicates which degrees of freedom are actually measured, i.e. the $r$ sensor locations.

The parameters to be identified are the eigenvalues (or modes) $\mu_{i}$ and mode shapes $\psi_{i}$ of system (1), which comprise the modal parameters, and are solutions of

$$
\left(\mu_{i}^{2} \mathcal{M}+\mu_{i} C+\mathcal{K}\right) \Psi_{i}=0, \quad \psi_{i}=L \Psi_{i}
$$


For simplicity, the measured outputs are displacements in model (1). However, also velocities, accelerations and, in general, any kind of sensor measurements fitting some linear system modeling can be used [3], yielding the same set of eigenvalues and mode shapes.

Sampling model (1) at rate $1 / \tau$ yields the discrete time state space model

$$
\left\{\begin{aligned}
x_{k+1} & =A x_{k}+v_{k} \\
y_{k} & =C x_{k}+w_{k}
\end{aligned}\right.
$$

with the states $x_{k}=\left[x(k \tau)^{T} \quad \dot{x}(k \tau)^{T}\right]^{T} \in \mathbb{R}^{n}$, the outputs $y_{k}=y(k \tau) \in \mathbb{R}^{r}$ and the unobserved input and output disturbances $v$ and $w$. The matrices $A \in \mathbb{R}^{n \times n}$ and $C \in \mathbb{R}^{r \times n}$ are the state transition and observation matrices, respectively, with

$$
A=\mathrm{e}^{\mathcal{L} \tau}, \text { where } \mathcal{L}=\left[\begin{array}{cc}
0 & I \\
-\mathcal{M}^{-1} \mathcal{K} & -\mathcal{M}^{-1} C
\end{array}\right], \quad C=\left[\begin{array}{ll}
L & 0
\end{array}\right] .
$$

The external force $v(t)$ and thus the state noise $\left(v_{k}\right)$ in model (3) can be non-stationary and colored noise [4, 24]. Define $r^{(\mathrm{ref})}$ as the number of so-called projection channels or reference sensors with $r^{\text {(ref })} \leq r$, which are a subset of the $r$ sensors and can be used for reducing the size of the matrices in the identification process [3].

The eigenstructure $(\lambda, \varphi)$ of system (3) is defined by the eigenvalues and eigenvectors of $A$ and by $C$ :

$$
\left(A-\lambda_{i} I\right) \phi_{i}=0, \quad \varphi_{i}=C \phi_{i}
$$

The desired modal parameters in (2) are equivalently found in the eigenstructure $(\lambda, \varphi)$ of (3) and it holds

$$
\mathrm{e}^{\mu_{i} \tau}=\lambda_{i}, \quad \psi_{i}=\varphi_{i} .
$$

The modal frequencies $f_{i}$ and damping coefficients $\xi_{i}$ are recovered directly from the eigenvalues $\lambda_{i}$ by

$$
f_{i}=\frac{\sqrt{a_{i}^{2}+b_{i}^{2}}}{2 \pi \tau}, \quad \xi_{i}=\frac{-100 b_{i}}{\sqrt{a_{i}^{2}+b_{i}^{2}}},
$$

where $a_{i}=\left|\arctan \mathfrak{J}\left(\lambda_{i}\right) / \mathfrak{R}\left(\lambda_{i}\right)\right|$ and $b_{i}=\ln \left|\lambda_{i}\right|, \mathfrak{R}(\cdot)$ and $\mathfrak{J}(\cdot)$ denoting the real and imaginary part of a complex variable, respectively.

Thus, vibration analysis is stated as the problem of identifying the eigenstructure of a linear dynamic system. Parameters of interest are the natural frequencies $f_{i}$, damping ratios $\xi_{i}$ and mode shapes $\varphi_{i}$.

\subsection{The general SSI algorithm}

There are many stochastic subspace identification algorithms in the literature, which differ in the construction of a matrix $\mathcal{H}$ from the data, from which the observability matrix is obtained. See e.g. [1-4] and the related references for an overview. Two examples are given in Appendix A. They all fit in the following general framework for the identification of the system matrices $A$ and $C$ of system (3).

Let the parameters $p$ and $q$ be given such that $p r \geq q r^{(\text {ref })} \geq n$. A matrix $\mathcal{H} \in \mathbb{R}^{(p+1) r \times q r^{(\text {ref })}}$ is built from the output data according to the chosen subspace algorithm, which will be called subspace matrix in the following. The subspace algorithm is chosen such that the corresponding subspace matrix enjoys (asymptotically for a large number of samples) the factorization property

$$
\mathcal{H}=O Z
$$

into the matrix of observability

$$
O \stackrel{\text { def }}{=} O(C, A) \stackrel{\text { def }}{=}\left[\begin{array}{c}
C \\
C A \\
\vdots \\
C A^{p}
\end{array}\right] \in \mathbb{R}^{(p+1) r \times n}
$$

and a matrix $\mathcal{Z}$ depending on the selected subspace algorithm. 
The observability matrix $O$ is obtained from a thin SVD of the matrix $\mathcal{H}$ and its truncation at the desired model order $n$ :

$$
\begin{aligned}
\mathcal{H} & =U \Sigma V^{T} \\
& =\left[\begin{array}{ll}
U_{1} & U_{0}
\end{array}\right]\left[\begin{array}{cc}
\Sigma_{1} & 0 \\
0 & \Sigma_{0}
\end{array}\right]\left[\begin{array}{l}
V_{1}^{T} \\
V_{0}^{T}
\end{array}\right], \\
\mathcal{O} & =U_{1} \Sigma_{1}^{1 / 2},
\end{aligned}
$$

where

$$
U_{1}=\left[\begin{array}{lll}
u_{1} & \ldots & u_{n}
\end{array}\right] \in \mathbb{R}^{(p+1) r \times n}, \quad V_{1}=\left[\begin{array}{lll}
v_{1} & \ldots & v_{n}
\end{array}\right] \in \mathbb{R}^{q r^{(\mathrm{ret})} \times n}, \quad \Sigma_{1}=\operatorname{diag}\left\{\sigma_{1}, \ldots, \sigma_{n}\right\} \in \mathbb{R}^{n \times n} .
$$

The observation matrix $C$ is then found in the first block-row of the observability matrix $O$. The state transition matrix $A$ is obtained from the shift invariance property of $O$, namely as the least squares solution of

$$
O^{\uparrow} A=O^{\downarrow} \text {, where } O^{\uparrow} \stackrel{\text { def }}{=}\left[\begin{array}{c}
C \\
C A \\
\vdots \\
C A^{p-1}
\end{array}\right], O^{\downarrow} \stackrel{\operatorname{def}}{=}\left[\begin{array}{c}
C A \\
C A^{2} \\
\vdots \\
C A^{p}
\end{array}\right] \text {. }
$$

The least squares solution can be obtained from

$$
A=O^{\uparrow} O^{\downarrow}
$$

where $^{\dagger}$ denotes the Moore-Penrose pseudoinverse. Note that the matrices $O$ (from (9)), $A$ and $C$ are identified up to an invertible matrix change of basis matrix $T$, such that the observability matrix $O T$ and the system matrices $C T$ and $T^{-1} A T$ may be identified. The eigenstructure $(\lambda, \varphi)$ in (4) is invariant under this change of basis.

\section{Multi-setup stochastic subspace identification}

The problem of stochastic subspace identification using non-simultaneously recorded data from multiple sensor setups was addressed in $[11,12]$ and generalized in [13]. Instead of a single record for the output $\left(y_{k}\right)$ of the system (3), $N_{s}$ records

$$
\underbrace{\left(\begin{array}{c}
y_{k}^{(1, \text { ref })} \\
y_{k}^{(1, \text { mov })}
\end{array}\right)}_{\text {Record 1 }} \underbrace{\left(\begin{array}{c}
y_{k}^{(2, \text { ref })} \\
y_{k}^{(2, \text { mov })}
\end{array}\right)}_{\text {Record 2 }} \cdots \underbrace{\left(\begin{array}{c}
y_{k}^{\left(N_{s}, \text { ref }\right)} \\
y_{k}^{\left(N_{s}, \text { mov }\right)}
\end{array}\right)}_{\text {Record } N_{s}}
$$

are now available collected successively. Each record $j$ contains data $y_{k}^{(j, \text { ref })}$ of dimension $r^{(\text {ref })}$ from a fixed reference sensor pool, and data $y_{k}^{(j \text { mov })}$ of dimension $r^{(j)}$ from a moving sensor pool. To each record $j=1, \ldots, N_{s}$ corresponds a state-space realization in the form [11-13]

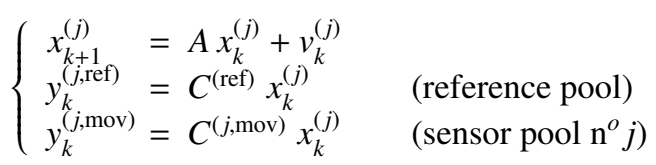

with a single state transition matrix $A$, since the same system is being observed ${ }^{1}$. The observation matrix $C^{(\text {ref })}$ with respect to the reference sensors is independent of the measurement setup as the reference sensors are fixed throughout

\footnotetext{
${ }^{1}$ This assumption may not strictly hold in practice e.g. due to temperature variations between the measurements [25], but is necessary to obtain meaningful global system identification results of the same structure. The following merging approach for system identification was successfully tested on a number of real structures [14-17] where small variations between the measurements naturally happen.
} 
the measurements, while the observation matrices $C^{(j, \mathrm{mov})}$ correspond to the moving sensor pool of each setup $j$. The global observation matrix is then defined as

$$
C=\left[\begin{array}{c}
C^{(\mathrm{ref})} \\
C^{(1, \mathrm{mov})} \\
\vdots \\
C^{\left(N_{s}, \mathrm{mov}\right)}
\end{array}\right],
$$

which contains information of all sensor positions.

In order to identify the eigenstructure of system (13) on the basis of the measurements (12), the output-only subspace algorithm of Section 2.2 is adapted. It is the aim to merge the data from the different records prior to the identification step of the algorithm, so that the system identification steps in Section 2.2 as well as the subsequent eigenstructure identification in (4)-(5) only have to be done once.

Local subspace matrices $\mathcal{H}^{(j)}$ can be computed on each of the $N_{s}$ data records in (12), which all yield factorization property (6) with a different observability matrix $O^{(j)}$ on the left and in general a different matrix $\mathcal{Z}^{(j)}$ on the right side. These matrices need to be merged somehow for a global merging approach, which would be easy if the matrices $\mathcal{Z}^{(j)}$ were equal for all setups $j$. However, there are several obstacles [13]:

- In general, the matrices $\mathcal{Z}^{(j)}$ are dependent on different sensor sets. However, this can be solved with referencebased subspace identification when using the same reference sensors in all setups.

- For most subspace algorithms, e.g. the data-driven algorithms in [2] as UPC, the matrices $\mathcal{Z}^{(j)}$ have no expected value as their size depends on the number of samples $N$ and the initial state $x_{0}^{(j)}$, or they are not uniquely defined due to LQ decompositions as in (A.4).

- The matrices $O^{(j)}$ and $\mathcal{Z}^{(j)}$ might be in a different modal basis for each setup $j$, so that in fact $\widehat{O}^{(j)} T_{j}$ and $T_{j}^{-1} \widehat{\mathcal{Z}}^{(j)}$ are identified with an invertible unknown change of basis matrix $T_{j} \in \mathbb{R}^{n \times n}$. This is also the reason why the global observation matrix (14) cannot be obtained straightforward from a separate system identification of the setups $j=1, \ldots, N_{s}$.

- Multi-setup measurements take place over a longer time period than a single measurement and it is likely that properties of the ambient excitation change between the measurement campaigns. Then, the covariance $Q^{(j)}$ of the excitation noise $v_{k}^{(j)}$ may change for the different records. As $\mathcal{Z}^{(j)}$ also depends on $Q^{(j)}$, it can be different for each record.

In [13] an algorithm is derived that takes these considerations into account. A global observability matrix $O^{\text {(all) }} \stackrel{\text { def }}{=}$ $O(C, A)($ cf. (7)) from all the records (12) is constructed as follows:

(a) For each setup $j$, the local subspace matrix $\mathcal{H}^{(j)}$ is built according to the chosen subspace algorithm using data $y_{k}^{(j, \text { ref })}$ and $y_{k}^{(j, \text { mov })}$, such that $\mathcal{H}^{(j)}$ fulfills factorization property (6) with observability matrix $O\left(\left[\begin{array}{lll}C^{(\mathrm{ref}) T} & C^{(j, \text { mov }) T}\end{array}\right]^{T}, A\right)$.

(b) Obtain the observability matrix $O^{(j)}$ from an SVD of $\mathcal{H}^{(j)}$, which is truncated at the desired model order $n$ as in (8)-(9). It holds

$$
O^{(j)}=O\left(\left[\begin{array}{c}
C^{(\mathrm{ref})} \\
C^{(j, \mathrm{mov})}
\end{array}\right], A\right) T_{j}
$$

with an unknown change of basis matrix $T_{j}$, as the decomposition of $\mathcal{H}^{(j)}$ is not unique.

(c) Select $O^{(j, \text { ref })}$ and $O^{(j, \text { mov })}$ from the block rows of $O^{(j)}$ as

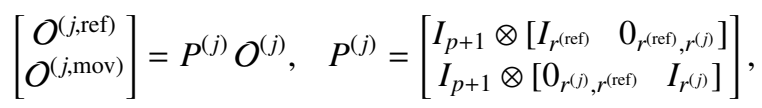

where $\otimes$ denotes the Kronecker product, $I_{a}$ is identity matrix of size $a \times a$ and $0_{a \times b}$ is the $a \times b$ zero matrix. Note that $O^{(j, \text { ref })}=O\left(C^{(\text {ref })}, A\right) T_{j}$ and $O^{(j, \text { mov })}=O\left(C^{(j, \text { mov })}, A\right) T_{j}$. 
(d) Set $O^{\text {(ref) }} \stackrel{\text { def }}{=} O^{(1, \text { ref) }}$ and compute the observability matrix parts

$$
\bar{O}^{(j, \text { mov })} \stackrel{\text { def }}{=} O^{(j, \text { mov })} O^{(j, \text { ref }) \dagger} O^{(\text {ref })},
$$

which are in the same modal basis. It holds $\bar{O}^{(j, \text { mov })}=O\left(C^{(j, \mathrm{mov})}, A\right)$.

(e) Each of the matrices $O^{\text {(ref) }}$ and $\bar{O}^{(j, m o v)}, j=1, \ldots, N_{s}$, are observability matrices of the form (7) with the block rows $C^{(\text {ref })} A^{i}$ and $C^{(j, \text { mov })} A^{i}$, for $i=0, \ldots, p$ respectively. Stacking these block rows in the order $C^{(\text {ref })} A^{i}, C^{(1, \text { mov })} A^{i}, \ldots, C^{\left(N_{s}, \text { mov }\right)} A^{i}$ for $i=0, \ldots, p$ leads to the global observability matrix

$$
O^{(\text {all })}=P\left[\begin{array}{c}
O^{(\text {ref })} \\
\bar{O}^{(1, \text { mov })} \\
\vdots \\
\bar{O}^{\left(N_{s}, \text { mov }\right)}
\end{array}\right] \text {, where } P=\left[\begin{array}{c}
P_{0} \\
P_{1} \\
\vdots \\
P_{p}
\end{array}\right] \text { with } P_{i}=\left[\begin{array}{cccc}
P_{i}^{(\text {ref }} & & & \\
& P_{i}^{(1)} & & \\
& & \ddots & \\
& & & P_{i}^{\left(N_{s}\right)}
\end{array}\right]
$$

and $P_{i}^{(j)}=\left[\begin{array}{lll}0_{r^{(j)}, i r^{(j)}} & I_{r^{(j)}} & 0_{r^{(j)},(p-i) r^{(j)}}\end{array}\right]$. Matrix $P$ is a permutation matrix.

Remark 1. Due to factorization property (6), step (d) of the merging algorithm is equivalent to

$$
\bar{O}^{(j, \text { mov })} \stackrel{\text { def }}{=} \mathcal{H}^{(j, \text { mov })} \mathcal{H}^{(j, \text { ref }) \dagger} O^{(\text {ref })},
$$

where $\mathcal{H}^{(j, \mathrm{mov})}$ and $\mathcal{H}^{(j, \mathrm{ref})}$ are defined by

$$
\left[\begin{array}{c}
\mathcal{H}^{(j, \mathrm{ref})} \\
\mathcal{H}^{(j, \mathrm{mov})}
\end{array}\right]=P^{(j)} \mathcal{H}^{(j)}
$$

analogously to $O^{(j, \text { ref })}$ and $O^{(j, m o v)}$.

Finally, the global system matrices $A$ and $C$ are obtained from $O^{(\text {all })}$ in one run. From $O^{(\text {all })}$, the global observation matrix $C$ is recovered as the first block row and the state transition matrix $A$ is the least squares solution of $O^{(\text {all }) \uparrow} A=$ $O^{(\text {all }) \downarrow}$.

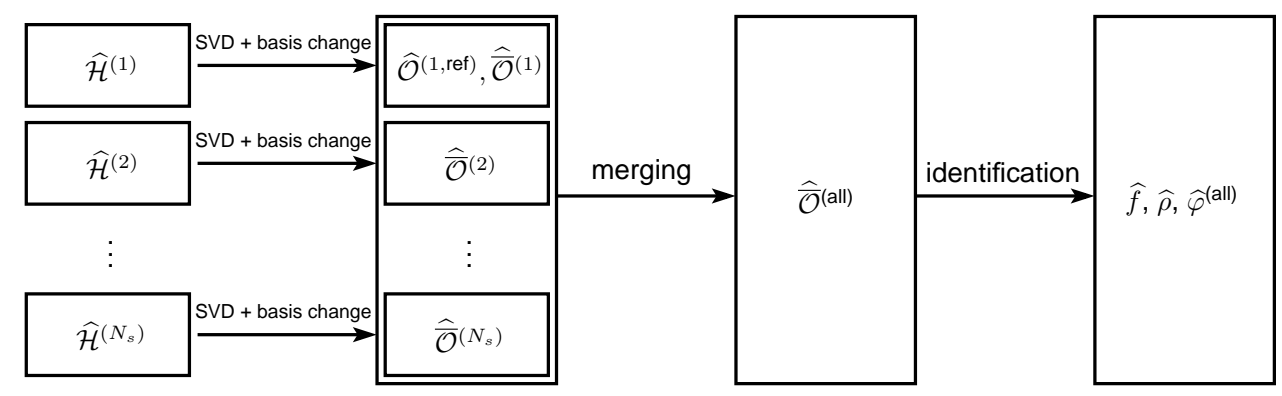

Figure 1: Merging and system identification scheme for modal analysis of multiple sensor setups.

\subsection{Modular iterative computation of the system matrices}

Matrices $A$ and $C$ can also be obtained in a modular way setup by setup: With (16) and defining the permutation matrix $\tilde{P}$ analogously to (16) but with $\tilde{P}_{i}^{(j)}=\left[\begin{array}{lll}0_{r^{(j)}, i r^{(j)}} & I_{r^{(j)}} & 0_{r^{(j)},(p-i-1) r^{(j)}}\end{array}\right]$ only for $i=0, \ldots, p-1$, the least squares 
solution for $A$ can be expressed as

$$
\begin{aligned}
& A=\left(O^{(\text {all }) \uparrow T} O^{(\text {all }) \uparrow}\right)^{-1} O^{(\text {all }) \uparrow T} O^{(\text {all }) \downarrow} \\
& =\left(\left[\tilde{P}\left[\begin{array}{c}
O^{(\mathrm{ref}) \uparrow} \\
\bar{O}^{(1, \mathrm{mov}) \uparrow} \\
\vdots \\
\bar{O}^{\left(N_{s}, \mathrm{mov}\right) \uparrow}
\end{array}\right]\right)^{T} \tilde{P}\left[\begin{array}{c}
O^{(\mathrm{ref}) \uparrow} \\
\bar{O}^{(1, \mathrm{mov}) \uparrow} \\
\vdots \\
\bar{O}^{\left(N_{s}, \mathrm{mov}\right) \uparrow}
\end{array}\right]\right)^{-1}\left(\tilde{P}\left[\begin{array}{c}
O^{(\mathrm{ref}) \uparrow} \\
\bar{O}^{(1, \mathrm{mov}) \uparrow} \\
\vdots \\
\bar{O}^{\left(N_{s}, \mathrm{mov}\right) \uparrow}
\end{array}\right]\right)^{T} \tilde{P}\left[\begin{array}{c}
O^{(\mathrm{ref}) \downarrow} \\
\bar{O}^{(1, \mathrm{mov}) \downarrow} \\
\vdots \\
\bar{O}^{\left(N_{s}, \mathrm{mov}\right) \downarrow}
\end{array}\right] \\
& =\left(O^{(\mathrm{ref}) \uparrow T} O^{(\mathrm{ref}) \uparrow}+\sum_{j=1}^{N_{s}} \bar{O}^{(j, \mathrm{mov}) \uparrow T} \bar{O}^{(j, \mathrm{mov}) \uparrow}\right)^{-1}\left(O^{(\mathrm{ref}) \uparrow T} O^{(\mathrm{ref}) \downarrow}+\sum_{j=1}^{N_{s}} \bar{O}^{(j, \mathrm{mov}) \uparrow T} \bar{O}^{(j, \mathrm{mov}) \downarrow}\right)
\end{aligned}
$$

as $\tilde{P}^{T} \tilde{P}=I$. Thus, the solution for $A$ can be computed iteratively setup by setup by starting with $K=O^{(\text {ref }) \uparrow T} O^{(\text {ref }) \uparrow}$, $L=O^{(\text {ref }) \uparrow T} O^{(\text {ref }) \downarrow}$, and updating $K=K+\bar{O}^{(j, \text { mov }) \uparrow T} \bar{O}^{(j, \text { mov }) \uparrow}, L=L+\bar{O}^{(j, \text { mov }) \uparrow T} \bar{O}^{(j, \text { mov }) \downarrow}$ in each setup $j$, from where at the end $A=K^{-1} L$ follows. Matrix $C$ can be directly filled with the first block rows of $O^{(\text {ref })}$ and $\bar{O}^{(j, \text { mov })}, j=$ $1, \ldots, N_{s}$, while the setups are processed. Like this, there is no need to assemble the potentially large matrix $O^{(\text {all) }}$ in (16) explicitly, but one can operate directly on the matrices $\bar{O}^{(j \text { mov })}$ that are obtained in each setup for the iterative computation of $A$ and $C$.

\subsection{Comparison to other merging approaches}

Processing the multi-setup measurement data is often achieved by performing the modal parameter identification for each record separately, and then merging the results obtained for records corresponding to different sensor setups. However, this procedure may require significant user input for the analysis of stabilization diagrams, especially when many setups are processed $[8,15]$. Mode matching between the different may be not easy in practice for closely spaced modes or modes that are weakly excited in some of the setups.

An empirical merging approach for covariance-driven subspace identification was described in [8], in which the output correlations of the different setups are combined before doing the global system identification, but without doing a normalization step as in (17) before the identification. While this approach has shown good results in several test cases [8-10] and no user interaction to match modes of different setups is necessary, it is lacking some theoretical properties when the ambient excitation changes between the measurements of the different setups. Then, Hankel matrices are combined with different left factors (due to changing sensor sets) and with different right factors (due to changing excitation). For example, assume two setups with $\mathcal{H}^{(1)}=O^{(1)} \mathcal{Z}^{(1)}$ and $\mathcal{H}^{(2)}=O^{(2)} \mathcal{Z}^{(2)}$, where $O^{(1)}$ and $O^{(2)}$ are in the same state space basis. Then, the matrices $\mathcal{Z}^{(1)}$ and $\mathcal{Z}^{(2)}$ can be different due to changes in the excitation, and the stacked global matrix

$$
\mathcal{H}^{(\text {all })}=\left[\begin{array}{l}
\mathcal{H}^{(1)} \\
\mathcal{H}^{(2)}
\end{array}\right]=\left[\begin{array}{l}
O^{(1)} \mathcal{Z}^{(1)} \\
\boldsymbol{O}^{(2)} \mathcal{Z}^{(2)}
\end{array}\right]
$$

does not have a theoretically valid factorization property for the extraction of the observability matrix for the subspace identification anymore: $O^{(1)}$ and $O^{(2)}$ cannot be extracted from $\mathcal{H}^{(\text {all) }}$ if $\mathcal{Z}^{(1)} \neq \mathcal{Z}^{(2)}$. Nonetheless, a matrix is obtained from an SVD of $\mathcal{H}^{\text {(all) }}$ that is considered as the observability matrix in the empirical approach, and the parts in the obtained mode shapes corresponding to each setup are rescaled in a postprocessing step with the help of the reference sensors [8] to account for possible changes in the excitation. While this approach may still be feasible for moderate changes in the unmeasured excitation characteristics, the considered merging approach in this paper is designed for taking into account these changes, which was proven in [13]. It combines the practical advantages of the empirical approach in [8] with a theoretical foundation. Furthermore, it applies to a large range of subspace methods, including data-driven subspace methods. Also, the merging approach can be used in an iterative scheme where no global subspace or observability matrix is necessary, but the desired system matrices can be computed iteratively setup by setup as pointed out in Section 3.1, which is a computational advantage if the number of setups is large.

Other global merging approaches in frequency domain were proposed for maximum likelihood identification in [26] and for NExT-ERA in [27, 28]. For subspace identification, the considered merging approach in this paper has been extended to frequency domain in [29]. 


\section{Covariance computation for stochastic subspace identification of a single setup}

In this section, the notations and basic principles of the covariance computations in the subsequent sections are introduced. In particular, the concept of perturbation to compute uncertainty bounds is defined and the covariance computation of the modal parameters from [23] is recalled.

\subsection{Definitions}

First, the notation of perturbation is defined. Let $X$ be a smooth and bounded matrix function of an artificial scalar variable, where $X(0)$ is the "true value" of $X$ and $X(t)$ is a perturbed (estimated) value of $X$, where $t$ is small. Thus, the matrix can be expressed as $X=X(t)$ when computed from an estimated $X$, while $X(0)$ is their true but unknown value. Using the Taylor expansion

$$
X(t)=X(0)+t \dot{X}(0)+O\left(t^{2}\right)
$$

a first-order perturbation is denoted by $\Delta X \stackrel{\text { def }}{=} t \dot{X}(0)=X(t)-X(0)+O\left(t^{2}\right)$ for small $t$. Then, a perturbation on $X$ can be propagated for any function $Y=f(X)$ by $\operatorname{vec}(\Delta Y)=\mathcal{J}_{Y, X} \operatorname{vec}(\Delta X)$, where $\mathcal{J}_{Y, X}$ is the sensitivity of vec $(Y)$ with respect to $\operatorname{vec}(X)$ and where vec denotes the vectorization operator, which stacks the columns of a matrix on top of each other. Finally, the covariance of $\operatorname{vec}(Y)$ is linked to the covariance of $\operatorname{vec}(X)$ by

$$
\operatorname{cov}(\operatorname{vec}(Y))=\mathcal{J}_{Y, X} \operatorname{cov}(\operatorname{vec}(X)) \mathcal{J}_{Y, X}^{T}
$$

For example, a first-order perturbation of the subspace matrix $\mathcal{H}$ is propagated to a perturbation of the system matrices $A$ and $C$ and to the modal parameters $f_{i}, \xi_{i}$ and $\varphi_{i}$ by deriving the respective sensitivities. The covariance of the subspace matrix $\mathcal{H}$ is denoted by $\Sigma_{\mathcal{H}} \stackrel{\text { def }}{=} \operatorname{cov}(\operatorname{vec}(\mathcal{H}))$. Its estimate $\widehat{\Sigma}_{\mathcal{H}}$ can be obtained from cutting the available sensor data into blocks and is explained in Appendix B.1.

\subsection{Covariances of modal parameters from single-setup SSI}

Consider the stochastic subspace identification from Section 2.2, where the modal parameters (natural frequencies $f_{i}$, damping ratios $\xi_{i}$ and mode shapes $\varphi_{i}$ ) are obtained from output-only data of one measurement setup. A firstorder perturbation on the subspace matrix $\mathcal{H}$ can be propagated to perturbations in the system matrices and finally to perturbations in the modal parameters. The variances of the modal parameters can be obtained in this way and depend on the covariance $\Sigma_{\mathcal{H}}$ of the subspace matrix. Thus, this offers a possibility to compute the uncertainty bounds of the modal parameters without repeating the system identification. In [23], this algorithm was described in detail for the covariance-driven SSI. A perturbation $\Delta A$ and $\Delta C$ of the system matrices $A$ and $C$ is connected to a perturbation of the subspace matrix through a sensitivity matrix $\mathcal{J}_{A C, \mathcal{H}}$ by

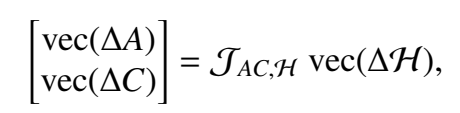

where $\mathcal{J}_{A C, \mathcal{H}}$ is recalled from [23] in Appendix B.2. Hence, the covariance $\Sigma_{A, C}$ of the vectorized system matrices can be expressed as

$$
\Sigma_{A, C} \stackrel{\text { def }}{=} \operatorname{cov}\left(\left[\begin{array}{l}
\operatorname{vec}(A) \\
\operatorname{vec}(C)
\end{array}\right]\right)=\mathcal{J}_{A C, \mathcal{H}} \Sigma_{\mathcal{H}} \mathcal{J}_{A C, \mathcal{H}}^{T} .
$$

As the modal parameters are functions of the system matrices $A$ and $C$, a perturbation analysis yields

$$
\Delta f_{i}=\mathcal{J}_{f_{i}, A} \operatorname{vec}(\Delta A), \quad \Delta \xi_{i}=\mathcal{J}_{\xi_{i}, A} \operatorname{vec}(\Delta A), \Delta \varphi_{i}=\mathcal{J}_{\varphi_{i}, A C}\left[\begin{array}{c}
\operatorname{vec}(\Delta A) \\
\operatorname{vec}(\Delta C)
\end{array}\right],
$$

where $\mathcal{J}_{f_{i}, A}, \mathcal{J}_{\xi_{i}, A}$ and $\mathcal{J}_{\varphi_{i}, A C}$ are the respective sensitivities for each mode $i$ and are stated in Appendix B.3. Finally, the covariances of the modal parameters are obtained as

$$
\begin{aligned}
\operatorname{cov}\left(\left[\begin{array}{l}
f_{i} \\
\xi_{i}
\end{array}\right],\left[\begin{array}{l}
f_{j} \\
\xi_{j}
\end{array}\right]\right) & =\left[\begin{array}{ll}
\mathcal{J}_{f_{i}, A} & 0_{1, r n} \\
\mathcal{J}_{\xi_{i}, A} & 0_{1, r n}
\end{array}\right] \Sigma_{A, C}\left[\begin{array}{cc}
\mathcal{J}_{f_{j}, A} & 0_{1, r n} \\
\mathcal{J}_{\xi_{j}, A} & 0_{1, r n}
\end{array}\right]^{T}, \\
\operatorname{cov}\left(\left[\begin{array}{c}
\mathfrak{R}\left(\varphi_{i}\right) \\
\mathfrak{J}\left(\varphi_{i}\right)
\end{array}\right],\left[\begin{array}{c}
\mathfrak{R}\left(\varphi_{j}\right) \\
\mathfrak{J}\left(\varphi_{j}\right)
\end{array}\right]\right) & =\left[\begin{array}{c}
\mathfrak{R}\left(\mathcal{J}_{\varphi_{i}, A C}\right) \\
\mathfrak{J}\left(\mathcal{J}_{\varphi_{i}, A C}\right)
\end{array}\right] \Sigma_{A, C}\left[\begin{array}{c}
\mathfrak{R}\left(\mathcal{J}_{\varphi_{j}, A C}\right) \\
\mathfrak{J}\left(\mathcal{J}_{\varphi_{j}, A C}\right)
\end{array}\right]^{T} .
\end{aligned}
$$




\section{Covariance computation for multi-setup stochastic subspace identification}

As the system matrices $A$ and $C$ are obtained differently for multi-setup measurements in Section 3 than for a single measurement in Section 2.2, their covariance computation has to be adapted. In the previous section, their covariance was obtained by deriving sensitivities of the system matrices with respect to the subspace matrix $\mathcal{H}$. Now, the global system matrices $A$ and $C$ depend on subspace matrices $\mathcal{H}^{(j)}$ from different measurement setups $j=1, \ldots, N_{s}$ and the respective sensitivities need to be derived. As the involved matrices can get very large for many setups and sensors, special care is taken to avoid their size explosion when obtaining the covariance $\Sigma_{A, C}$ for the multi-setup measurements in this section. After this step, the covariance of the modal parameters can be obtained from $\Sigma_{A, C}$ in (23).

The covariance computation for the multi-setup case is done analogously to the single-setup case, but following the computation in Section 3. First, perturbations $\Delta \mathcal{H}^{(j)}$ of the local subspace matrices are propagated to perturbations $\Delta O^{\text {(ref) }}$ and $\Delta \bar{O}^{(j, \mathrm{mov})}$ of the normalized parts of the merged observability matrix $O^{\text {(all) }}$ in (16), and second, these perturbations are propagated to perturbations $\Delta A$ and $\Delta C$ in the global system matrices. Finally, the covariances $\Sigma_{A, C}$ of the vectorized system matrices are computed.

The results of these derivations are summarized in this section, while the respective sensitivity matrices are derived in detail in Appendix C.1.

Lemma 2. Small perturbations in $\mathcal{H}^{(j)}$ are propagated to $O^{(\mathrm{ref})}$ and $\bar{O}^{(j, \mathrm{mov})}, j=1, \ldots, N_{s}$, by

$$
\begin{aligned}
\operatorname{vec}\left(\Delta O^{(\mathrm{refl})}\right) & =\mathcal{J}_{O^{\text {(ref) }}, \mathcal{H}^{(1)} \operatorname{vec}\left(\Delta \mathcal{H}^{(1)}\right),} \\
\operatorname{vec}\left(\Delta \bar{O}^{(j, \mathrm{mov})}\right) & =\mathcal{J}_{\bar{O}^{j}, O_{\text {(ref) }}} \mathcal{J}_{O^{\text {(ref) }}, \mathcal{H}^{(1)} \operatorname{vec}\left(\Delta \mathcal{H}^{(1)}\right)+\mathcal{J}_{\bar{O}^{j}, \mathcal{H}^{(j)}} \operatorname{vec}\left(\Delta \mathcal{H}^{(j)}\right),}
\end{aligned}
$$

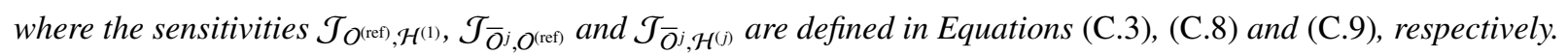

Proof. See Appendix C.1.

The perturbations in $O^{(\mathrm{ref})}$ and $\bar{O}^{(j, \mathrm{mov})}, j=1, \ldots, N_{s}$, are propagated to $A$ and $C$ in the following lemma, based on the solution for the system matrices in the multi-setup setting in (19).

Lemma 3. Small perturbations in $O^{(\mathrm{ref})}$ and $\bar{O}^{(j, \mathrm{mov})}, j=1, \ldots, N_{s}$, are propagated to $A$ and $C$ by

$$
\begin{aligned}
& \operatorname{vec}(\Delta A)=\mathcal{J}_{A, O^{(\text {ref })}} \operatorname{vec}\left(\Delta O^{(\text {ref })}\right)+\sum_{j=1}^{N_{s}} \mathcal{J}_{A, \bar{O}^{j}} \operatorname{vec}\left(\Delta \bar{O}^{(j, \mathrm{mov})}\right), \\
& \operatorname{vec}(\Delta C)=\mathcal{J}_{C, O^{(\text {ref })} \operatorname{vec}\left(\Delta O^{(\mathrm{ref})}\right)+\sum_{j=1}^{N_{s}} \mathcal{J}_{C, \bar{O}^{j}} \operatorname{vec}\left(\Delta \bar{O}^{(j, \mathrm{mov})}\right)},
\end{aligned}
$$

where the sensitivities $\mathcal{J}_{A, O^{\text {(ref) }},} \mathcal{J}_{A, \bar{O}^{j}}, \mathcal{J}_{C, O^{\text {(ref) }}}$ and $\mathcal{J}_{C, \bar{O}^{j}}$ are defined in Equations (C.12), (C.13) and (C.14), respectively.

\section{Proof. See Appendix C.1.}

Thus, a perturbation of the system matrices is the linear combination of the perturbations of all local (normalized) observability matrices. Plugging the results of Lemma 2 into Lemma 3, the perturbations of the system matrices can be expressed by the perturbations of the local subspace matrices and it follows

$$
\left[\begin{array}{c}
\operatorname{vec}(\Delta A) \\
\operatorname{vec}(\Delta C)
\end{array}\right]=\sum_{j=1}^{N_{s}} \mathcal{J}_{A C, j} \operatorname{vec}\left(\Delta \mathcal{H}^{(j)}\right)=\left[\begin{array}{lll}
\mathcal{J}_{A C, 1} & \ldots & \mathcal{J}_{A C, N_{s}}
\end{array}\right]\left[\begin{array}{c}
\operatorname{vec}\left(\Delta \mathcal{H}^{(1)}\right) \\
\vdots \\
\operatorname{vec}\left(\Delta \mathcal{H}^{\left(N_{s}\right)}\right)
\end{array}\right]
$$

where

$$
\begin{aligned}
& \mathcal{J}_{A C, 1} \stackrel{\text { def }}{=}\left[\begin{array}{l}
\mathcal{J}_{A, O^{\text {(ref) }}} \\
\mathcal{J}_{C, O^{\text {(ref) }}}
\end{array}\right] \mathcal{J}_{O^{\text {(ref) }}, \mathcal{H}^{(1)}}+\sum_{j=1}^{N_{s}}\left[\begin{array}{l}
\mathcal{J}_{A, \bar{O}^{j}} \\
\mathcal{J}_{C, \bar{O}^{j}}
\end{array}\right] \mathcal{J}_{\bar{O}^{j}, O^{\text {(ref) }}} \mathcal{J}_{O^{\text {(ref) }}, \mathcal{H}^{(1)},} \\
& \mathcal{J}_{A C, j} \stackrel{\text { def }}{=}\left[\begin{array}{l}
\mathcal{J}_{A, \bar{O}^{j}} \\
\mathcal{J}_{C, \bar{O}^{j}}
\end{array}\right] \mathcal{J}_{\bar{O}^{j}, \mathcal{H}^{(j)}}, \quad j \geq 2 .
\end{aligned}
$$


From (24), it follows for the covariance $\Sigma_{A, C}$ of the vectorized system matrices

$$
\Sigma_{A, C}=\operatorname{cov}\left(\left[\begin{array}{c}
\operatorname{vec}(A) \\
\operatorname{vec}(C)
\end{array}\right]\right)=\left[\begin{array}{lll}
\mathcal{J}_{A C, 1} & \ldots & \mathcal{J}_{A C, N_{s}}
\end{array}\right] \operatorname{cov}\left(\left[\begin{array}{c}
\operatorname{vec}\left(\mathcal{H}^{(1)}\right) \\
\vdots \\
\operatorname{vec}\left(\mathcal{H}^{\left(N_{s}\right)}\right)
\end{array}\right]\right)\left[\begin{array}{c}
\mathcal{J}_{A C, 1}^{T} \\
\vdots \\
\mathcal{J}_{A C, N_{s}}^{T}
\end{array}\right]
$$

However, this expression is dependent on the covariance of the stacked vectorized subspace matrices of all measurement setups, which grows quadratically with the number of setups and sensors and easily causes memory problems. In the following theorem, a more efficient solution is proposed.

Theorem 4. Let $\Sigma_{\mathcal{H}^{(j)}}=\operatorname{cov}\left(\operatorname{vec} \mathcal{H}^{(j)}\right), j=1, \ldots, N_{s}$, be the covariances of the local subspace matrices and let the sensitivities in (25) be given. Then, the covariance of the system matrices obtained from multi-setup SSI writes as

$$
\Sigma_{A, C}=\sum_{j=1}^{N_{s}} \mathcal{J}_{A C, j} \Sigma_{\mathcal{H}^{(j)}} \mathcal{J}_{A C, j}^{T}
$$

Proof. As the data records from different measurement setups are collected at different times, they can be assumed to be uncorrelated. Hence, the local subspace matrices $\mathcal{H}^{(j)}$ are statistically independent and it holds $\operatorname{cov}\left(\operatorname{vec} \mathcal{H}^{\left(j_{1}\right)}, \operatorname{vec} \mathcal{H}^{\left(j_{2}\right)}\right)=$ 0 for $j_{1} \neq j_{2}$. Thus, it follows from (24)

$$
\Sigma_{A, C}=\left[\begin{array}{lll}
\mathcal{J}_{A C, 1} & \cdots & \mathcal{J}_{A C, N_{s}}
\end{array}\right]\left[\begin{array}{ccc}
\Sigma_{\mathcal{H}^{(1)}} & & 0 \\
& \ddots & \\
0 & & \Sigma_{\mathcal{H}^{\left(N_{s}\right)}}
\end{array}\right]\left[\begin{array}{c}
\mathcal{J}_{A C, 1}^{T} \\
\vdots \\
\mathcal{J}_{A C, N_{s}}^{T}
\end{array}\right]
$$

and the assertion follows.

Note that in Theorem 4, the size of the involved covariance matrices is reduced considerably by assuming statistical independence of the data from different setups, as only the matrices $\Sigma_{\mathcal{H}^{(j)}}, j=1, \ldots, N_{s}$, are needed and no crosscovariance estimates between different setups.

Using Theorem 4, the covariance and hence the uncertainty bounds of the modal parameters can be computed as stated in (23).

\section{Mode shape normalization}

As the mode shapes are only defined up to a complex-valued factor, a normalization may be chosen when evaluating their covariance. In [23] the so-called unit modal displacement is chosen, where the mode shape is scaled such that one element is unity. In this case, the mode shape $\varphi_{i}$ yields

$$
\varphi_{i}=\frac{1}{\left(C \phi_{i}\right)_{k}} C \phi_{i}
$$

for an eigenvector $\phi_{i}$ of $A$, where $\left(C \phi_{i}\right)_{k}$ indicates the $k$-th entry of the arbitrarily scaled mode shape $\left(C \phi_{i}\right)$. Thus, $\varphi_{i}$ is defined such that its $k$-th entry is 1 . The respective covariance computation for the resulting mode shape is derived in [23] and summarized in Appendix B.3.

However, this normalization scheme leads to a variance of zero of the $k$-the mode shape element, as its value is deterministic. Moreover, the $k$-th value of $\left(C \phi_{i}\right)$ needs to be clearly non-zero for normalization, thus leading to a mode shape value with a rather high amplitude but zero variance.

In the following, a more intuitive normalization scheme is proposed, where the resulting mode shape has its maximal amplitude of deflection, and the corresponding sensitivities for the mode shape covariance computation are derived. Like this, the mode shape is rotated such that the imaginary part of one mode shape component is zero. 
Proposition 5. Let $\phi_{i}$ be an eigenvector of $A$ and $k$ be the component of the corresponding mode shape $\left(C \phi_{i}\right)$ with the maximal absolute value. Denote this component by $s_{i, k} \stackrel{\text { def }}{=}\left(C \phi_{i}\right)_{k}=t_{i, k} \mathrm{e}^{\mathrm{i} \alpha_{i, k}}$, where $t_{i, k}$ and $\alpha_{i, k}$ are its absolute value and phase, respectively. Define the normalized mode shape as

$$
\widetilde{\varphi}_{i} \stackrel{\text { def }}{=} \mathrm{e}^{-\mathrm{i} \alpha_{i, k}} C \phi_{i}
$$

Then, a perturbation on the system matrices is propagated to the mode shape by

$$
\Delta \widetilde{\varphi}_{i}=\mathcal{J}_{\widetilde{\varphi}_{i}, A C}\left[\begin{array}{c}
\operatorname{vec}(\Delta A) \\
\operatorname{vec}(\Delta C)
\end{array}\right]
$$

where the sensitivity $\mathcal{J}_{\widetilde{\varphi}_{i}, A C}$ writes as

$$
\mathcal{J}_{\widetilde{\varphi}_{i}, A C}=\mathrm{e}^{-\mathrm{i} \alpha_{i, k}}\left(-\mathrm{i} t_{i, k}^{-2} C \phi_{i}\left[\begin{array}{ll}
-\mathfrak{J}\left(s_{i, k}\right) e_{k}^{T} & \mathfrak{R}\left(s_{i, k}\right) e_{k}^{T}
\end{array}\right]+\left[\begin{array}{ll}
I_{r} & \mathrm{i} I_{r}
\end{array}\right]\right)\left[\begin{array}{ll}
C \mathfrak{R}\left(\mathcal{J}_{\phi_{i}, A}\right) & \mathfrak{R}\left(\phi_{i}\right)^{T} \otimes I_{r} \\
C \mathfrak{J}\left(\mathcal{J}_{\phi_{i}, A}\right) & \mathfrak{J}\left(\phi_{i}\right)^{T} \otimes I_{r}
\end{array}\right],
$$

with the unit vector $e_{k} \in \mathbb{R}^{r}$ being 1 at entry $k$ and 0 elsewhere and $\mathcal{J}_{\phi_{i}, A}$ defined in Appendix B.3.

Proof. See Appendix C.2.

It is emphasized that the unit modal displacement normalization in (27) as well as the proposed normalization to the maximal amplitude of deflection are both theoretically valid normalization schemes, while introducing different constraints. They are applied after the global mode shape estimate is obtained from the considered multi-setup system identification, where the full mode shape is extracted at once with all its parts correctly scaled. The associated uncertainty computation inherits this property as well, and it is not necessary to normalize the mode shape at one of the reference sensors to unity. The mode shape normalization does not depend anymore on the choice of the reference sensors that stay fixed throughout all the setups. In the unit modal displacement scheme, a particular sensor has to be chosen for the mode shape normalization, which now can be any sensor, and the variance of the real and imaginary part of this point is thus 0 . In the new scheme, the mode shape is rotated by the phase angle of the degree of freedom with maximal absolute value, so that only the variance of the imaginary part of this point is 0 . Both approaches benefit from the global modular identification and uncertainty computation.

A summary of the computation of the modal parameters and their variances from multiple setups with the presented strategies is summarized in Algorithm 1, where matrices $A$ and $C$ are computed in one run. Alternatively to Step 12 in the algorithm, the iterative computation scheme from Section 3.1 can also be used.

\section{Application: Modal analysis of Z24 Bridge}

\subsection{The test case}

The proposed algorithms have been applied on vibration data of the Z24 Bridge [30], a benchmark of the COST F3 European network. The analyzed data is the response of the bridge to ambient excitation (traffic under the bridge) measured in 154 points, mainly in the vertical and at some points also the transverse and lateral directions, and sampled at $100 \mathrm{~Hz}$. Because at most 33 sensors were available (counting one sensor for each measured direction), $N_{s}=9$ data sets have been recorded, each containing the measurements from $r^{\text {(ref) }}=5$ fixed and 28 moving sensors, except dataset $j=5$ containing only 22 moving sensors. Like this, altogether 251 sensors were mimicked. Each signal contains 65,535 samples. In Figure 2, the model of the bridge with all sensor positions and directions is shown.

\subsection{Modal analysis and uncertainty computation}

In operational modal analysis, the true system order is in general unknown. A larger model order must be assumed in order to retrieve a desired number of modes, as weakly excited modes only appear at high model orders and the noise dynamics need to be decoupled from the system dynamics in case of colored excitation noise appearing in practice [31]. With system identification results at multiple model orders, the identified physical system modes can be distinguished from noise modes, as the latter tend to vary at different orders [3, 23, 32]. The identified frequencies are plotted in a stabilization diagram against their model order. From the modes common to many models and using 

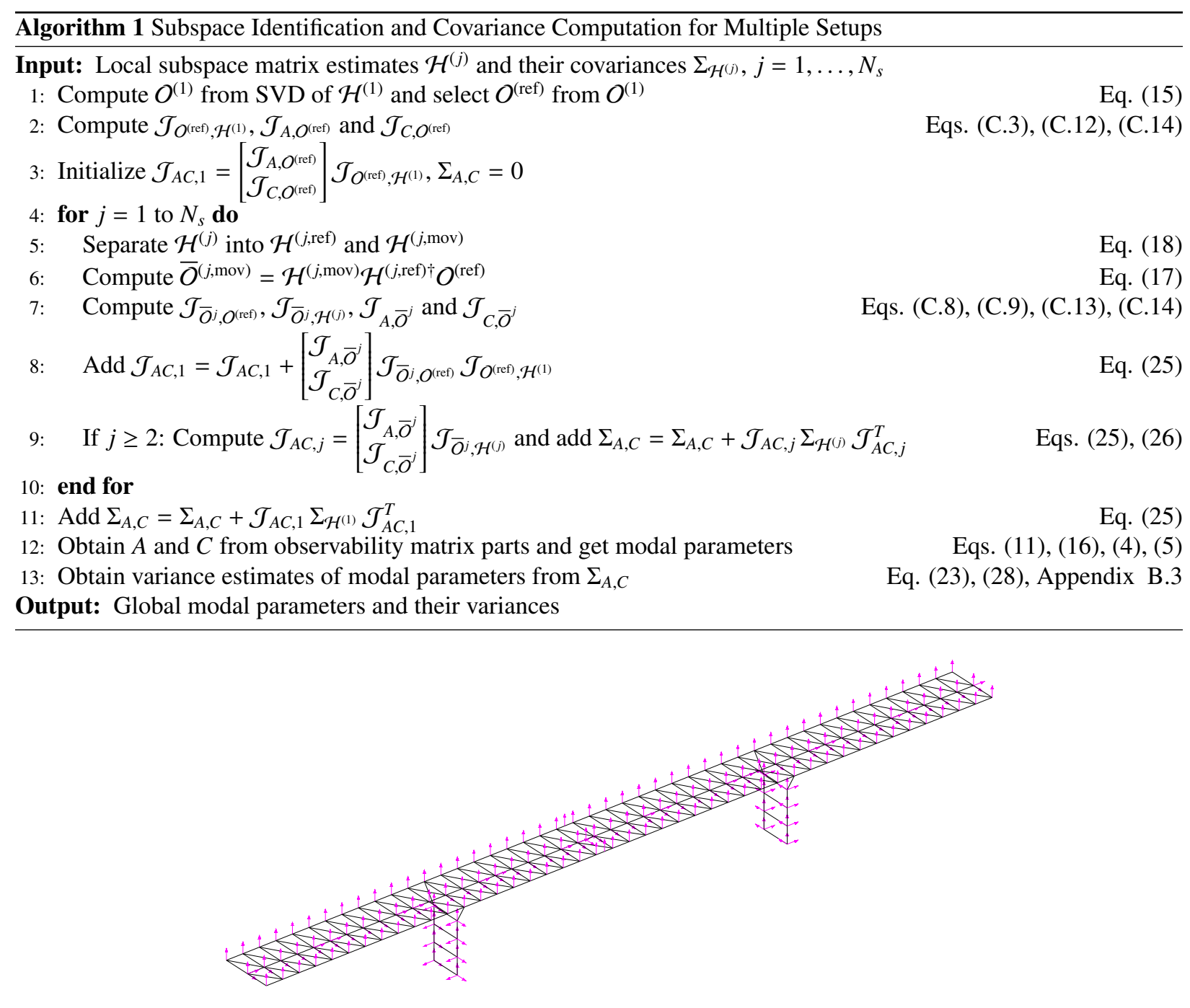

Figure 2: Model of Z24 Bridge with sensor positions and directions.

further stabilization criteria, such as a threshold on damping values, low variation between modes and mode shapes of successive orders etc., the final estimated model is obtained.

Using the reference-based covariance-driven subspace method (see Appendix A), the subspace matrices $\widehat{\mathcal{H}}^{(j)}$ are computed for each setup $j=1, \ldots, 9$ with the parameters $p+1=q=40$. The global observability matrix $\widehat{O}^{(\text {all })}$ is then obtained from the merging procedure explained in Section 3, which is used for the global system identification with the generic subspace identification algorithm and the subsequent computation of the modal parameters in Section 2. This identification is repeated while truncating at the model orders $n=1, \ldots, 100$.

The data of each setup $j=1, \ldots, 9$ was cut into $n_{b}=200$ blocks for the estimation of $\Sigma_{\mathcal{H}^{(j)}}$ of each setup for the covariance computations (see Appendix B.1). Then, the standard deviations of the system identification results are obtained from the covariance computation for multi-setup SSI in Section 5 at each of the model orders $n=1, \ldots, 100$. In Figure 3, the stabilization diagram at these model orders containing the natural frequencies and their standard deviations is shown. Note that the uncertainty bounds on the estimated modal parameters can be used as another criterion for the selection of the modes in a stabilization diagram. In Figure 3, only frequencies are plotted with the criteria $f_{i}<30 \mathrm{~Hz}, 0.1<\xi_{i}<10$ and a threshold on the coefficient of variation of the frequencies as $100 \sigma_{f_{i}} / f_{i}<1.5$ 


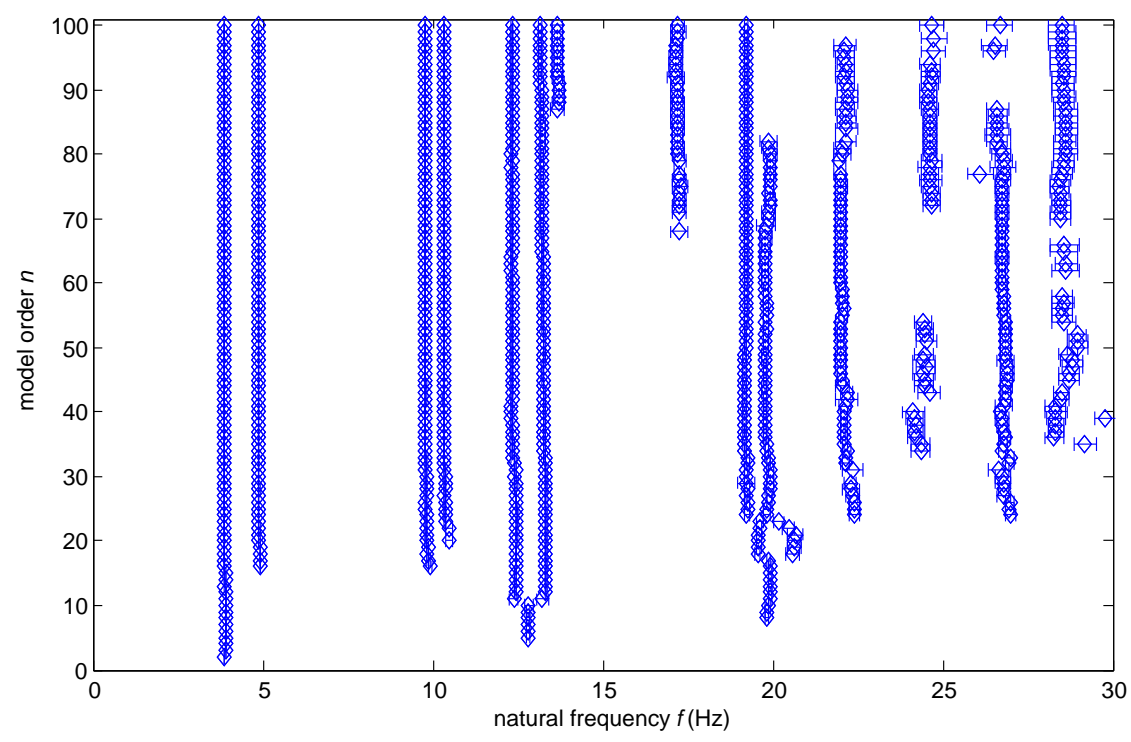

Figure 3: Stabilization diagram of natural frequencies with confidence intervals $\pm \sigma_{f_{i}}$.

Table 1: Identified frequencies and damping ratios with coefficients of variation.

\begin{tabular}{c||cc|cc} 
Mode & $f_{i}(\mathrm{~Hz})$ & $100 \sigma_{f_{i}} / f_{i}$ & $\xi_{i}(\%)$ & $100 \sigma_{\xi_{i}} / \xi_{i}$ \\
\hline 1 & 3.864 & 0.05 & 0.61 & 8.8 \\
2 & 4.883 & 0.05 & 1.43 & 4.7 \\
3 & 9.787 & 0.06 & 1.36 & 3.6 \\
4 & 10.32 & 0.09 & 1.87 & 5.4 \\
5 & 12.33 & 0.19 & 3.40 & 4.9 \\
6 & 13.27 & 0.16 & 3.47 & 4.3 \\
7 & 17.20 & 0.95 & 7.18 & 14 \\
8 & 19.22 & 0.21 & 2.83 & 8.6 \\
9 & 19.79 & 0.23 & 3.97 & 5.5 \\
10 & 26.83 & 0.58 & 4.86 & 13 \\
\hline
\end{tabular}

(less than $1.5 \%$ ), where $\sigma_{f_{i}}=\sqrt{\operatorname{cov}\left(f_{i}\right)}$.

From the stabilization diagram, 10 modes could be identified. Note that in the frequency range of $20-30 \mathrm{~Hz}$ a number of modes appear that seem to stabilize in the diagram, where only the mode at nearly $27 \mathrm{~Hz}$ is a true structural mode. In Table 1, the natural frequencies and damping ratios of all modes are summarized together with their coefficients of variation in percent of the respective value. In Figure 4, the real parts of the estimated mode shapes at their maximal amplitude are presented. The obtained system identification results are in good accordance with previous studies and the entire set of all 10 modes could be retrieved from the ambient vibration data in this study, e.g. in comparison to $[26,33]$ where some of the modes could only be retrieved from shaker data.

In Figures 5 and 6, the $\pm 2 \sigma_{\varphi_{i}}$ confidence bounds of the mode shapes are shown, where the mode shape normalization from Section 6 is used. Only one sensor row is used for the visualization, namely the front row (see Figure 2) for vertical and torsional modes in Figure 5 and the middle row with the sensors in the lateral direction for lateral mode 2 in Figure 6. As it can be seen from these figures as well as from Table 1, only mode 7 has quite high confidence bounds, as it is apparently weakly excited and appears only late in the stabilization diagram. In general, the uncertainty bounds of the frequencies are much smaller than those of damping ratios, which is coherent with statistical theory [34]. 
mode 1

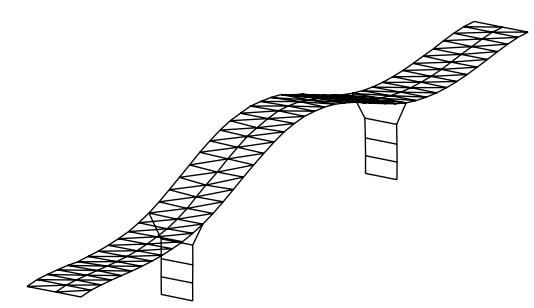

mode 4

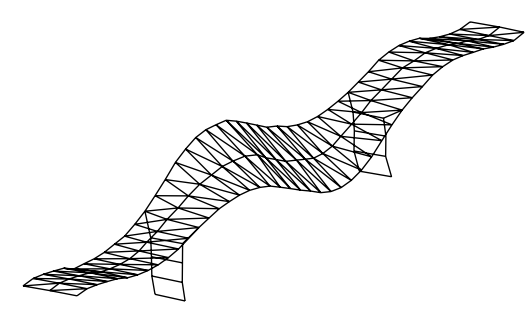

mode 7

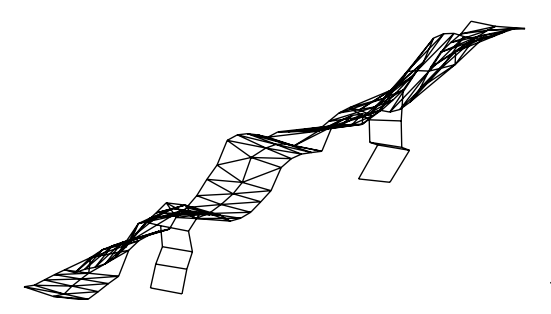

mode 2

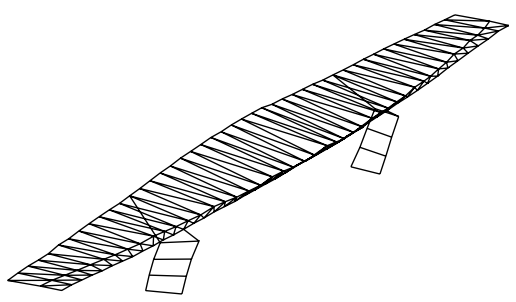

mode 5

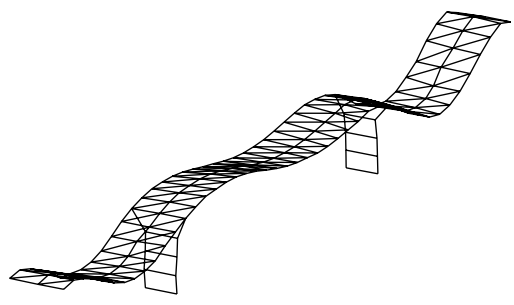

mode 8

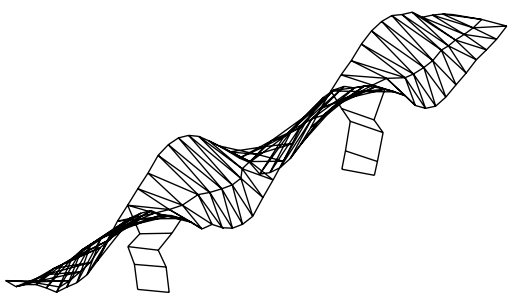

mode 3

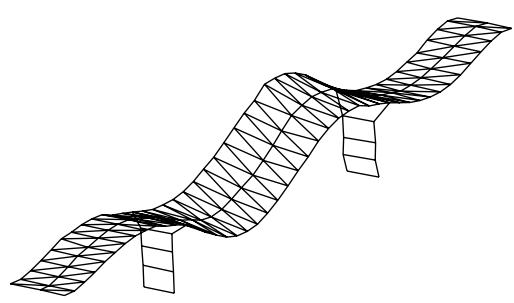

mode 6

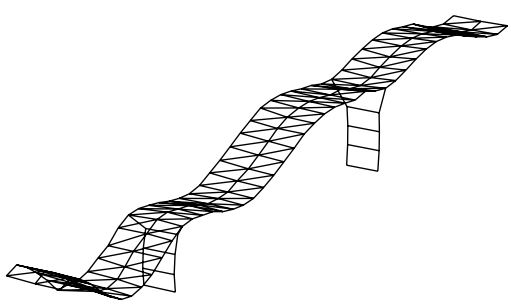

mode 9
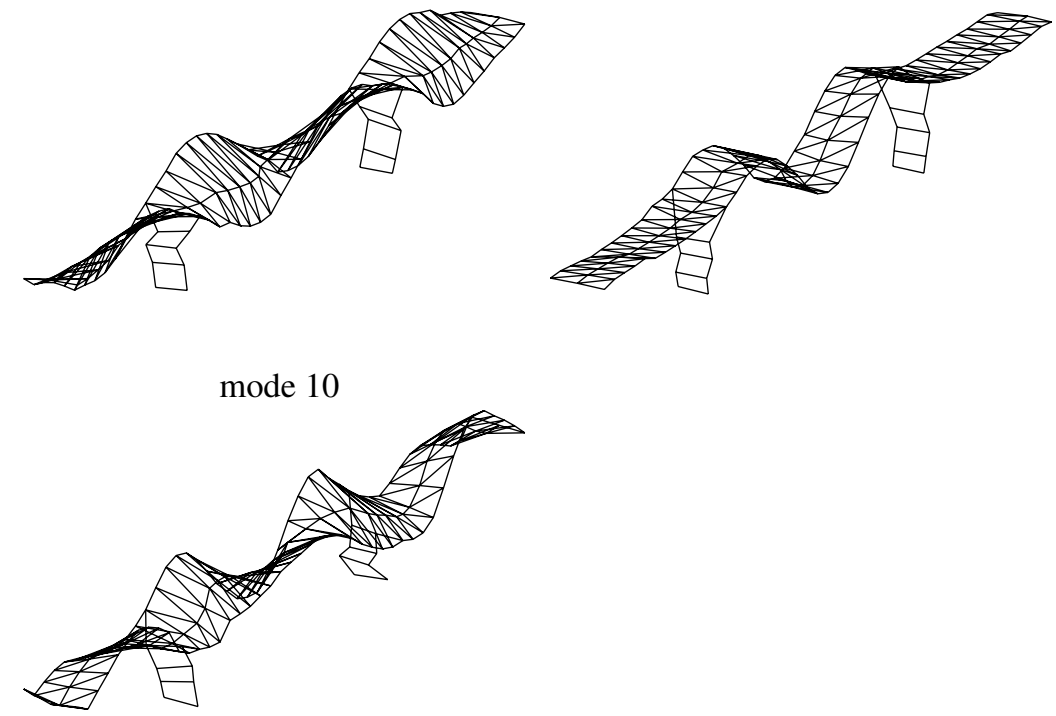

Figure 4: Mode shapes of Z24 Bridge.

Finally, the confidence bounds for the mode shape normalization from Section 6 are compared to the unit modal displacement normalization from [23] exemplarily for the first mode shape in Figure 7, where also the coefficients of variation in each degree of freedom are shown. In (a), the proposed mode shape normalization from Section 6 is used, where the mode shape is normalized to its maximum amplitude of deflection. In (b)-(d) the unit modal displacement normalization from [23] is used with respect to different sensors: (b) w.r.t. first reference sensor; (c) w.r.t. sensor in the middle peak; (d) w.r.t. sensor in the smaller side peak. In all normalization schemes, the coefficient of variation of the mode shape parts with high amplitude are lower than of the parts close to zero, as one expects. The coefficients of variation of the mode shapes are quite different depending on the chosen normalization scheme. For example, the coefficient of variation in the middle peak varies between $2-2.5 \%$ in schemes (a), (b) and (d), while it is 0 in (c). In the side peaks, they vary between $0-20 \%$, reaching up to $600 \%$ at the degrees of freedom close to zero. Note that the 
mode 1

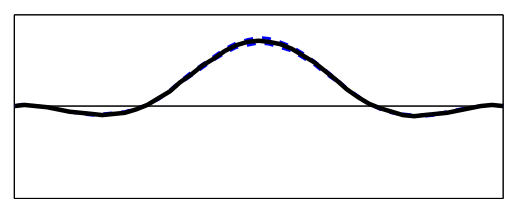

mode 5

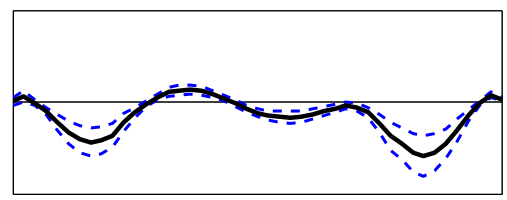

mode 8

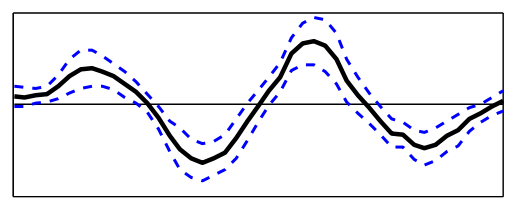

mode 3

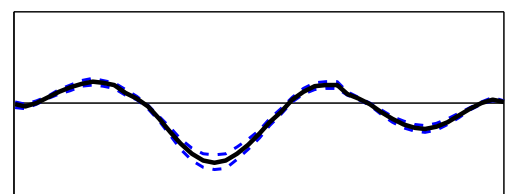

mode 6

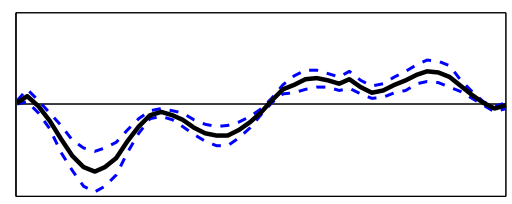

mode 9

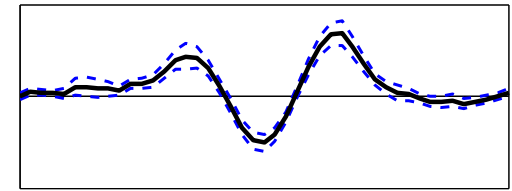

mode 4

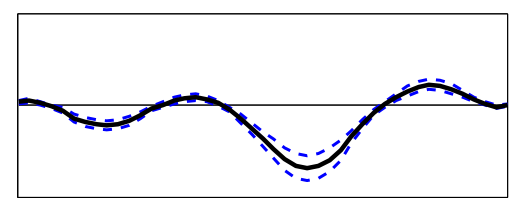

mode 7

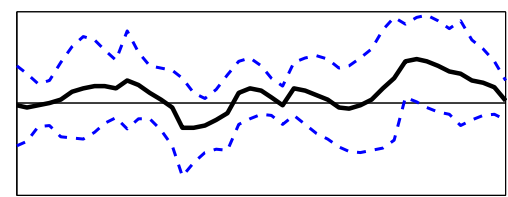

mode 10

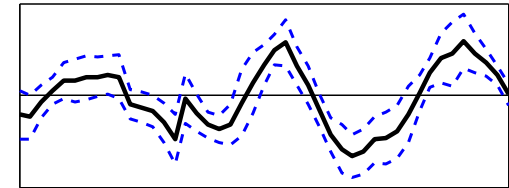

Figure 5: Mode shapes at sensors of the front row of the bridge deck in vertical direction with $\pm 2 \sigma_{\varphi_{i}}$ confidence bound.

mode 2

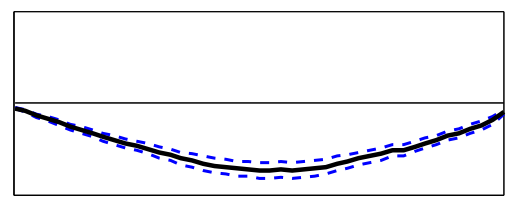

Figure 6: Mode shape at sensors of the middle row of the bridge deck in lateral direction with $\pm 2 \sigma_{\varphi_{i}}$ confidence bound

covariance computation with the new scheme in (a) is less influenced by the choice of a particular sensor for the mode shape normalization, in constrast to (b)-(d).

(a)

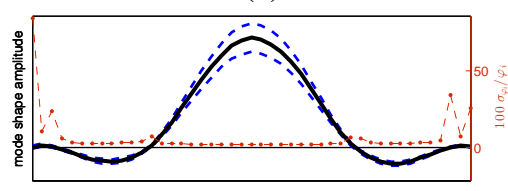

(b)

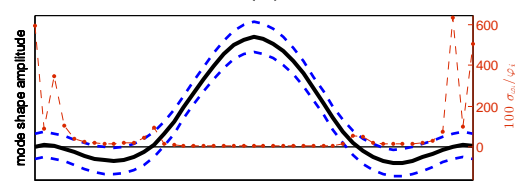

(c)

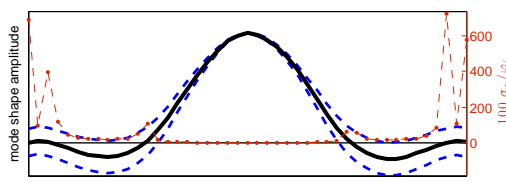

(d)

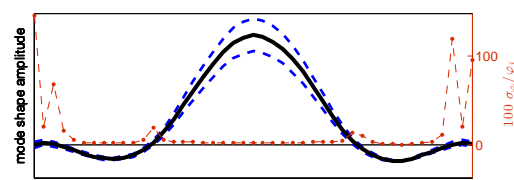

Figure 7: Mode shape normalization of first mode with strategy from Section 6 in (a) and with strategy from [23] in (b)-(d), where the normalization sensors are: (b) first reference sensor, (c) sensor in middle peak, (d) sensor in right side peak. The $\pm 6 \sigma_{\varphi_{i}}$ confidence bound of the mode shape and the coefficients of variation in $\%$ are shown. 


\section{Discussion and conclusions}

In this paper, a memory efficient algorithm has been derived for the uncertainty quantification of modal parameters, which are obtained from a theoretically profound merging algorithm for multi-setup subspace identification [13]. The design of the algorithm takes possibly different ambient excitation properties between the setups into account, while being scalable for a large number of setups and sensors. The theoretical properties of the merging algorithm were shown in [13] and are extended to the new associated uncertainty computation described in this paper. It has been shown that the covariance of the system matrices is a weighted sum of the covariances of all local subspace matrices for each setup, which can be computed efficiently and iteratively. A new intuitive normalization scheme for the mode shape covariance computation has been proposed. The method was successfully applied on multi-setup ambient vibration data of the Z24 Bridge benchmark. The proposed method is applicable to any kind of covariance-driven subspace methods including frequency domain subspace methods as in [29], as well as data-driven methods [35], provided a scheme for the covariance computation of the subspace matrix is available.

The numerical results obtained in Section 7 are comparable to similar previous studies [8, 23, 36], still exhibiting a more automated and parameter free multi-setup approach since only one global identification step is performed as well as only one global related uncertainty computation for all setups. No matching of modes between setups is needed anymore. In the stabilization diagrams, the confidence bounds vary with the model order and can also be a tool for selecting modes of high quality as well as discriminating between spurious and physical modes. Weakly excited modes tend to have high confidence intervals, which is not surprising. These observations still have to be validated by more experiments.

In terms of efficiency, the multi-setup covariance computation consumes less memory and is also computationally more efficient than working on the full subspace matrix built from all the measurements, which would yield a practically infeasible computation for real cases. Avoiding the computation of big matrices allows also to go high in the model order, which is useful for analyzing weakly excited modes that appear late in the stabilization diagram. Moreover, Theorem 4 ensures that the method will scale with more setups and sensors accordingly with little penalty. Computation time is expected to increase linearly with the number of setups. The computation time for the reported application (identification and covariance computation) was around 15 minutes on a desktop computer, but optimization was not the topic of interest for this paper and may be investigated in the future.

Since the multi-setup SSI retrieves the mode shape estimates using only one global identification step and avoids gluing mode shape parts from different setups, there are less constraints on the normalization of the mode shape for its covariance computation with the proposed scheme in this paper. In particular, the choice of the mode shape component for normalization in (27) in the unit modal displacement weighting from [23] (leading to zero variance of this component) can be done on any sensor, not only one of the reference sensors available in all setups. With the new scheme in Section 6, the mode shape is normalized to maximal amplitude, and the mode shape components with a low amplitude show also a low absolute uncertainty and vice versa. The coefficients of variation of the components with higher amplitude are the lowest as they are typically better estimated, while components close to zero show huge coefficients of variation. Both normalization schemes are theoretically valid and can be used, while the new scheme follows a more intuitive approach.

Note that changes in the modal parameters between measurements are not considered in the merging approach, but may be present in reality e.g. due to temperature or other environmental variations. The effect of significant changes in the structure on the computation of modal parameters that are still meaningful in the multiple setup problem remains to be investigated, and the pertinence of a naive fusion of the modal parameters may even be questionable in this case. These variations are a general nuisance for system identification from multiple measurements, affecting no less the considered merging method in this paper than other approaches. While small changes between the measurements have not posed a problem for the considered method in [14-17], this problem as well as the effects on the uncertainty computation should be addressed in future works.

\section{Acknowledgment}

This support from the European projects FP7-PEOPLE-2009-IAPP 251515 ISMS is acknowledged. The data for this research were obtained in the framework of the BRITE-EURAM Programme CT96 0277, SIMCES and provided by the SAMCO organization. 


\section{Appendix A. Examples of SSI Algorithms}

In this section, examples of subspace matrices $\mathcal{H}$ are given that are related to two widely used output-only stochastic subspace identification algorithms. Let $N+p+q$ be the number of available samples and $y_{k}^{\text {(ref) }} \in \mathbb{R}^{r^{\text {(ref) }}}$ the vector containing the reference sensor data, which is a subset of $y_{k}$ for all samples. Then, define the data matrices

$$
\mathcal{Y}^{+}=\frac{1}{\sqrt{N}}\left[\begin{array}{cccc}
y_{q+1} & y_{q+2} & \vdots & y_{N+q} \\
y_{q+2} & y_{q+3} & \vdots & y_{N+q+1} \\
\vdots & \vdots & \vdots & \vdots \\
y_{q+p+1} & y_{q+p+2} & \vdots & y_{N+p+q}
\end{array}\right], \quad \mathcal{Y}^{-}=\frac{1}{\sqrt{N}}\left[\begin{array}{cccc}
y_{q}^{\text {(ref) }} & y_{q+1}^{\text {(ref) }} & \vdots & y_{N+q-1}^{(\mathrm{ref})} \\
y_{q-1}^{(\mathrm{ref})} & y_{q}^{(\mathrm{ref})} & \vdots & y_{N+q-2}^{(\mathrm{ref})} \\
\vdots & \vdots & \vdots & \vdots \\
y_{1}^{\text {(ref) }} & y_{2}^{\text {(ref) }} & \vdots & y_{N}^{\text {(ref) }}
\end{array}\right]
$$

For covariance-driven SSI $[1,3,4]$, let $R_{i} \stackrel{\text { def }}{=} \mathbf{E}\left(y_{k} y_{k-i}^{(\text {ref }) T}\right) \in \mathbb{R}^{r \times r^{\text {ref })}}$ and the block Hankel matrix

$$
\mathcal{H}^{\mathrm{cov}} \stackrel{\operatorname{def}}{=}\left[\begin{array}{cccc}
R_{1} & R_{2} & \ldots & R_{q} \\
R_{2} & R_{3} & \ldots & R_{q+1} \\
\vdots & \vdots & \ddots & \vdots \\
R_{p+1} & R_{p+2} & \ldots & R_{p+q}
\end{array}\right]
$$

be the theoretical output-correlation and subspace matrices, where $\mathbf{E}$ denotes the expectation operator. It enjoys the factorization property (6), where $\mathcal{Z}$ is the controllability matrix. The empirical correlations are estimated from $\widehat{R}_{i}=\frac{1}{N-i} \sum_{k=i+1}^{N} y_{k} y_{k-i}^{(\mathrm{ret}) T}$. Another estimate of the covariance-driven subspace matrix is

$$
\widehat{\mathcal{H}}^{\mathrm{cov}}=\boldsymbol{y}^{+}\left(\boldsymbol{y}^{-}\right)^{T} .
$$

For data-driven SSI with the Unweighted Principal Component (UPC) algorithm [2-4], the estimate of the subspace matrix is defined as

$$
\widehat{\mathcal{H}}^{\text {dat }}=\mathcal{Y}^{+}\left(\boldsymbol{y}^{-}\right)^{T}\left(\boldsymbol{y}^{-}\left(\boldsymbol{y}^{-}\right)^{T}\right)^{\dagger} \boldsymbol{y}^{-} .
$$

where $^{\dagger}$ denotes the pseudoinverse. Then, factorization property (6) holds asymptotically, where $\mathcal{Z}$ is the Kalman filter state matrix. With the partitioning of the thin LQ decomposition of

$$
\left[\begin{array}{l}
y^{-} \\
y^{+}
\end{array}\right]=\left[\begin{array}{cc}
R_{11} & 0 \\
R_{21} & R_{22}
\end{array}\right]\left[\begin{array}{l}
Q_{1} \\
Q_{2}
\end{array}\right]
$$

the relation $\widehat{\mathcal{H}}^{\text {dat }}=R_{21} Q_{1}$ follows, where $R_{21} \in \mathbb{R}^{(p+1) r \times q r^{\text {(ref) }}}$ and $Q_{1} \in \mathbb{R}^{q r^{\text {(ref })} \times N}$. As $Q_{1}$ is an orthogonal matrix, the estimate of the observability matrix $\widehat{O}$ is obtained from $R_{21}$ in the implementation of the algorithm, and $\widehat{\mathcal{H}}^{\text {dat, } \mathrm{R}} \stackrel{\text { def }}{=} R_{21}$ is defined as a subspace matrix.

\section{Appendix B. Details for covariance computation in Section 4}

Appendix B.1. Estimating the covariance of the subspace matrix

For an estimation of the covariance $\Sigma_{\mathcal{H}}$, the data matrices $\mathcal{Y}^{+}$and $\mathcal{Y}^{-}$from (A.1) are split into $n_{b}$ blocks and normalized with respect to their length, such that

$$
\sqrt{N} \boldsymbol{y}^{+}=\sqrt{N_{b}}\left[\begin{array}{llll}
y_{1}^{+} & y_{2}^{+} & \ldots & \mathcal{y}_{n_{b}}^{+}
\end{array}\right], \quad \sqrt{N} \boldsymbol{y}^{-}=\sqrt{N_{b}}\left[\begin{array}{llll}
y_{1}^{-} & y_{2}^{-} & \ldots & \boldsymbol{y}_{n_{b}}^{-}
\end{array}\right],
$$

where each block $\mathcal{Y}_{j}^{+}$and $\mathcal{Y}_{j}^{-}$may have the same length $N_{b}$, with $n_{b} \cdot N_{b}=N$ for simplicity. Each block may be long enough to assume statistical independence between the blocks. On each of theses blocks, the corresponding subspace matrix can be estimated and used for an empirical estimation of $\Sigma_{\mathcal{H}}$. The covariance of the subspace matrix in the covariance-driven case follows easily from the covariance of the sample mean and was used e.g. in [23]. There, the 
covariance of the vectorized Hankel matrix (A.2) is obtained from the covariance $\Sigma_{\mathcal{R}}$ of the vectorized correlations $\mathcal{R} \stackrel{\text { def }}{=}\left[\begin{array}{llll}R_{1}^{T} & R_{2}^{T} & \ldots & R_{p+q}^{T}\end{array}\right]^{T} \in \mathbb{R}^{(p+q) r \times r^{(\text {ref })}}$ as

$$
\Sigma_{\mathcal{H}^{\mathrm{cov}}}=S_{1} \Sigma_{\mathcal{R}} S_{1}^{T}
$$

to reduce the size of the involved matrices, where $S_{3} \in \mathbb{R}^{(p+1) r q r^{(\mathrm{ref})} \times(p+q) r r^{(\mathrm{ref})}}$ is a selection matrix that fills the vectorized Hankel matrix $\mathcal{H}^{\mathrm{cov}}$ with the vectorized correlations in $\mathcal{R}$ such that $\operatorname{vec}(\mathcal{H})=S_{3} \operatorname{vec}(\mathcal{R})$ and is defined as

$$
S_{1} \stackrel{\text { def }}{=}\left[\begin{array}{llll}
S_{1,1}^{T} & S_{1,2}^{T} & \ldots & S_{1, q}^{T}
\end{array}\right] \text { where } \quad S_{1, k} \stackrel{\text { def }}{=} I_{r^{\text {(ref) }}} \otimes\left[\begin{array}{lll}
0_{(p+1) r \times(k-1) r} & I_{(p+1) r} & 0_{(p+1) r \times(q-k) r)}
\end{array}\right] .
$$

Let $\widehat{\mathcal{R}}_{j}$ be an estimate of $\mathcal{R}$ computed on the data blocks $\mathcal{Y}_{j}^{+}$and $\mathcal{Y}_{j}^{-}$, then $\widehat{\mathcal{R}}=\frac{1}{n_{b}} \sum_{j=1}^{n_{b}} \widehat{\mathcal{R}}_{j}$ and a covariance estimate follows from

$$
\widehat{\Sigma}_{\mathcal{H}^{\text {cov }}}=S_{1} \widehat{\Sigma}_{\mathcal{R}} S_{1}^{T} \quad \text { where } \widehat{\Sigma}_{\mathcal{R}}=\frac{1}{n_{b}\left(n_{b}-1\right)} \sum_{j=1}^{n_{b}}\left(\operatorname{vec}\left(\widehat{\mathcal{R}}_{j}\right)-\operatorname{vec}(\widehat{\mathcal{R}})\right)\left(\operatorname{vec}\left(\widehat{\mathcal{R}}_{j}\right)-\operatorname{vec}(\widehat{\mathcal{R}})\right)^{T}
$$

Similarly, the estimate (A.3) of the covariance-driven subspace matrix can be used directly and a subspace matrix estimate $\widehat{\mathcal{H}}_{j}^{\text {cov }} \stackrel{\text { def }}{=} \mathcal{Y}_{j}^{+}\left(\mathcal{Y}_{j}^{-}\right)^{T}$ is computed on each data block from (B.1). Then, $\widehat{\mathcal{H}}^{\text {cov }}=\frac{1}{n_{b}} \sum_{j=1}^{n_{b}} \widehat{\mathcal{H}}_{j}^{\text {cov }}$ and a covariance estimate of the covariance-driven subspace matrix writes as

$$
\widehat{\Sigma}_{\mathcal{H}^{\text {cov }}}=\frac{1}{n_{b}\left(n_{b}-1\right)} \sum_{j=1}^{n_{b}}\left(\operatorname{vec}\left(\widehat{\mathcal{H}}_{j}^{\text {cov }}\right)-\operatorname{vec}\left(\widehat{\mathcal{H}}^{\mathrm{cov}}\right)\right)\left(\operatorname{vec}\left(\widehat{\mathcal{H}}_{j}^{\mathrm{cov}}\right)-\operatorname{vec}\left(\widehat{\mathcal{H}}^{\mathrm{cov}}\right)\right)^{T} .
$$

For the covariance estimation in the data-driven case, the non-uniqueness of the LQ decomposition (A.4) has to be taken into account. Estimates for the UPC algorithm have been derived in [35].

\section{Appendix B.2. Covariance estimation of the system matrices $A$ and $C$}

The covariance estimation of the matrices $A$ and $C$ is done in three steps: First, a perturbation $\Delta \mathcal{H}$ of the subspace matrix is propagated to a perturbation $\Delta O$ of the observability matrix, and second, a perturbation $\Delta O$ is propagated to perturbations $\Delta A$ and $\Delta C$ in the system matrices. Finally, the covariances of the vectorized system matrices are computed.

First, define the permutation

$$
\mathcal{P}_{a, b} \stackrel{\text { def }}{=} \sum_{k=1}^{a} \sum_{l=1}^{b} E_{k, l}^{a, b} \otimes E_{l, k}^{b, a},
$$

where $E_{k, l}^{a, b}$ is a matrix of size $a \times b$ that is equal to 1 at position $(k, l)$ and zero elsewhere. For any matrix $X \in \mathbb{R}^{a, b}$ it has the property [22]

$$
\operatorname{vec}\left(X^{T}\right)=\mathcal{P}_{a, b} \operatorname{vec}(X)
$$

Lemma 6 ([22]). Let $\sigma_{i}, u_{i}$ and $v_{i}$ be the ith singular value, left and right singular vector of some matrix $X \in \mathbb{R}^{a \times b}$ and $\triangle X$ a small perturbation on $X$. Then it holds

$$
\Delta \sigma_{i}=\left(v_{i} \otimes u_{i}\right)^{T} \operatorname{vec}(\Delta X), B_{i}\left[\begin{array}{c}
\Delta u_{i} \\
\Delta v_{i}
\end{array}\right]=C_{i} \operatorname{vec}(\Delta X)
$$

where

$$
B_{i} \stackrel{\text { def }}{=}\left[\begin{array}{cc}
I_{a} & -\frac{1}{\sigma_{i}} X \\
-\frac{1}{\sigma_{i}} X^{T} & I_{b}
\end{array}\right], \quad C_{i} \stackrel{\text { def }}{=} \frac{1}{\sigma_{i}}\left[\begin{array}{c}
v_{i}^{T} \otimes\left(I_{a}-u_{i} u_{i}^{T}\right) \\
\left(u_{i}^{T} \otimes\left(I_{b}-v_{i} v_{i}^{T}\right)\right) \mathcal{P}_{a, b}
\end{array}\right]
$$


Using this result for $X=\mathcal{H}$, the sensitivity of the observability matrix is derived in [23]. Define $S_{2} \stackrel{\text { def }}{=} \sum_{k=1}^{n} E_{(k-1) n+k, k}^{n^{2}, n}$ and it follows

where $\mathcal{J}_{O, \mathcal{H}} \in \mathbb{R}^{(p+1) r n \times(p+1) r q r^{(\mathrm{ref})}}$ with $^{2}$

$$
\operatorname{vec}(\Delta O)=\mathcal{J}_{O, \mathcal{H}} \operatorname{vec}(\Delta \mathcal{H})
$$

$$
\mathcal{J}_{O, \mathcal{H}} \stackrel{\text { def }}{=} \frac{1}{2}\left(I_{n} \otimes U_{1} \Sigma_{1}^{-1 / 2}\right) S_{2}\left[\begin{array}{c}
\left(v_{1} \otimes u_{1}\right)^{T} \\
\vdots \\
\left(v_{n} \otimes u_{n}\right)^{T}
\end{array}\right]+\left(\Sigma_{1}^{1 / 2} \otimes\left[\begin{array}{ll}
I_{(p+1) r} & 0_{(p+1) r \times q r} \text { (ref) }
\end{array}\right]\right)\left[\begin{array}{c}
B_{1}^{\dagger} C_{1} \\
\vdots \\
B_{n}^{\dagger} C_{n}
\end{array}\right] .
$$

In the next step, the sensitivity of the system matrices with respect to the observability matrix is obtained as

$$
\operatorname{vec}(\Delta A)=\mathcal{J}_{A, O} \operatorname{vec}(\Delta O), \quad \operatorname{vec}(\Delta C)=\mathcal{J}_{C, O} \operatorname{vec}(\Delta O),
$$

where $\mathcal{J}_{A, O} \in \mathbb{R}^{n^{2} \times(p+1) r n}, \mathcal{J}_{C, O} \in \mathbb{R}^{r n \times(p+1) r n}$, with

$$
\begin{aligned}
& \mathcal{J}_{A, O} \stackrel{\text { def }}{=}\left(I_{n} \otimes O^{\uparrow^{\dagger}} S_{\downarrow}\right)-\left(A^{T} \otimes O^{{ }^{\dagger}} S_{\uparrow}\right)+\left(\left(O^{\downarrow^{T}} S_{\downarrow}-A^{T} O^{{ }^{T}} S_{\downarrow}\right) \otimes\left(O^{\uparrow^{T}} O^{\uparrow}\right)^{-1}\right) \mathcal{P}_{(p+1) r, n}, \\
& \mathcal{J}_{C, O} \stackrel{\text { def }}{=} I_{n} \otimes\left[\begin{array}{ll}
I_{r} & 0_{r, p r}
\end{array}\right]
\end{aligned}
$$

and the selection matrices

$$
S_{\uparrow} \stackrel{\text { def }}{=}\left[\begin{array}{ll}
I_{p r} & 0_{r \times p r}
\end{array}\right], \quad S_{\downarrow} \stackrel{\text { def }}{=}\left[\begin{array}{ll}
0_{r \times p r} & I_{p r}
\end{array}\right],
$$

such that $S_{\uparrow} O=O^{\uparrow}, S_{\downarrow} O=O^{\downarrow}$. Thus, the sensitivity of the system matrices with respect to the subspace matrix $\mathcal{J}_{A C, \mathcal{H}}$ in (20)-(21) writes as

$$
\mathcal{J}_{A C, \mathcal{H}}=\left[\begin{array}{c}
\mathcal{J}_{A, O} \\
\mathcal{J}_{C, O}
\end{array}\right] \mathcal{J}_{O, \mathcal{H}}
$$

\section{Appendix B.3. Covariance estimation of the modal parameters}

In [23], the sensitivity derivations for the eigenvalues and eigenvectors of a matrix and subsequently for the modal parameters are stated, based on derivations in $[22,37]$. They are summarized in the following. Let $\lambda_{i}, \phi_{i}$ and $\chi_{i}$ be the $i$-th eigenvalue, right eigenvector and left eigenvector of $A$ with

$$
A \phi_{i}=\lambda_{i} \phi_{i}, \quad \chi_{i}^{*} A=\lambda_{i} \chi_{i}^{*},
$$

where $^{*}$ denotes the complex conjugate transpose. Then,

$$
\Delta \lambda_{i}=\mathcal{J}_{\lambda_{i}, A} \operatorname{vec}(\Delta A), \quad \Delta \phi_{i}=\mathcal{J}_{\phi_{i}, A} \operatorname{vec}(\Delta A),
$$

where $\mathcal{J}_{\lambda_{i}, A} \in \mathbb{C}^{1 \times n^{2}}, \mathcal{J}_{\phi_{i}, A} \in \mathbb{C}^{n \times n^{2}}$, with

$$
\mathcal{J}_{\lambda_{i}, A} \stackrel{\text { def }}{=} \frac{1}{\chi_{i}^{*} \phi_{i}}\left(\phi_{i}^{T} \otimes \chi_{i}^{*}\right), \quad \mathcal{J}_{\phi_{i}, A} \stackrel{\text { def }}{=}\left(\lambda_{i} I_{n}-A\right)^{\dagger}\left(\phi_{i}^{T} \otimes\left(I_{n}-\frac{\phi_{i} \chi_{i}^{*}}{\chi_{i}^{*} \phi_{i}}\right)\right) .
$$

Let furthermore $\tilde{\lambda}_{i} \stackrel{\text { def }}{=} \ln \left(\lambda_{i}\right) / \tau=\left(b_{i}+a_{i} \mathrm{i}\right) / \tau$ be the eigenvalue of the continuous-time state transition matrix corresponding to $A$ as in (5). Let the natural frequency $f_{i}$ and the damping ratio $\xi_{i}$ be given in (5), and suppose that the element $k$ of the mode shape $\varphi_{i}$ is scaled to unity, i.e. $\varphi_{i}=C \phi_{i} /\left(C \phi_{i}\right)_{k}$ [23]. Then,

$$
\Delta f_{i}=\mathcal{J}_{f_{i}, A} \operatorname{vec}(\Delta A), \quad \Delta \xi_{i}=\mathcal{J}_{\xi_{i}, A} \operatorname{vec}(\Delta A), \quad \Delta \varphi_{i}=\mathcal{J}_{\varphi_{i}, A C}\left[\begin{array}{c}
\operatorname{vec}(\Delta A) \\
\operatorname{vec}(\Delta C)
\end{array}\right],
$$

where $\mathcal{J}_{f_{i}, A}, \mathcal{J}_{\xi_{i}, A} \in \mathbb{R}^{1 \times n^{2}}, \mathcal{J}_{\varphi_{i}, A C} \in \mathbb{C}^{r \times\left(n^{2}+r n\right)}$, with

$$
\left[\begin{array}{c}
\mathcal{J}_{f_{i}, A} \\
\mathcal{J}_{\xi_{i}, A}
\end{array}\right] \stackrel{\text { def }}{=}\left[\begin{array}{c}
\mathcal{J}_{f_{i}, \lambda_{i}} \\
\mathcal{J}_{\xi_{i}, \lambda_{i}}
\end{array}\right]\left[\begin{array}{c}
\mathfrak{R}\left(\mathcal{J}_{\lambda_{i}, A}\right) \\
\mathfrak{J}\left(\mathcal{J}_{\lambda_{i}, A}\right)
\end{array}\right], \quad \mathcal{J}_{\varphi_{i}, A C} \stackrel{\text { def }}{=} \frac{1}{\left(C \phi_{i}\right)_{k}}\left(I_{r}-\left[\begin{array}{lll}
0_{r, k-1} & \varphi_{i} & 0_{r, r-k}
\end{array}\right]\right)\left[\begin{array}{ll}
C \mathcal{J}_{\phi_{i}, A} & \phi_{i}^{T} \otimes I_{r}
\end{array}\right],
$$

and

$$
\left[\begin{array}{c}
\mathcal{J}_{f_{i}, \lambda_{i}} \\
\mathcal{J}_{\xi_{i}, \lambda_{i}}
\end{array}\right] \stackrel{\text { def }}{=} \frac{1}{\tau\left|\lambda_{i}\right|^{2}\left|\tilde{\lambda}_{i}\right|}\left[\begin{array}{cc}
1 /(2 \pi) & 0 \\
0 & 100 /\left|\tilde{\lambda}_{i}\right|^{2}
\end{array}\right]\left[\begin{array}{cc}
\mathfrak{R}\left(\tilde{\lambda}_{i}\right) & \mathfrak{J}\left(\tilde{\lambda}_{i}\right) \\
-\mathfrak{J}\left(\tilde{\lambda}_{i}\right)^{2} & \mathfrak{R}\left(\tilde{\lambda}_{i}\right) \mathfrak{J}\left(\tilde{\lambda}_{i}\right)
\end{array}\right]\left[\begin{array}{cc}
\mathfrak{R}\left(\lambda_{i}\right) & \mathfrak{J}\left(\lambda_{i}\right) \\
-\mathfrak{J}\left(\lambda_{i}\right) & \mathfrak{R}\left(\lambda_{i}\right)
\end{array}\right] \in \mathbb{R}^{2 \times 2} .
$$

${ }^{2}$ Note that $\mathcal{J}_{O, \mathcal{H}}=\mathcal{B}+C$ with the notation of [23]. 


\section{Appendix C. Proofs}

\section{Appendix C.1. Proofs of Section 5}

Proof of Lemma 2. First, define the selection matrices

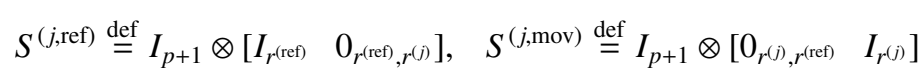

analogous to (15), which select the reference and moving sensor parts, respectively, from a local subspace matrix $\mathcal{H}^{(j)}$ or observability matrix $O^{(j)}$ when premultiplied.

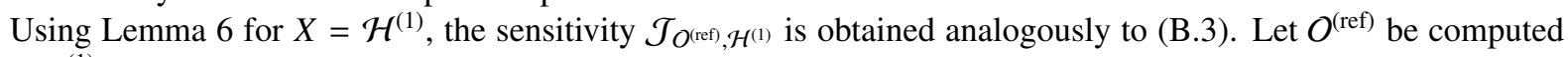
from $\mathcal{H}^{(1)}$ as described in steps (a)-(d) in Section 3, i.e.

$$
\mathcal{H}^{(1)}=\left[\begin{array}{ll}
U_{1} & U_{0}
\end{array}\right]\left[\begin{array}{cc}
\Sigma_{1} & 0 \\
0 & \Sigma_{0}
\end{array}\right]\left[\begin{array}{l}
V_{1}^{T} \\
V_{0}^{T}
\end{array}\right], \quad O^{(\text {ref })}=S^{(1, \text { ref })} U_{1} \Sigma_{1}^{1 / 2} .
$$

It follows analogously to (B.3)

$$
\begin{aligned}
\Delta O^{(\text {ref })} & =S^{(1, \text { ref) }} \Delta\left(U_{1}\right) \Sigma_{1}^{1 / 2}+S^{(1, \text { ref })} U_{1} \Delta\left(\Sigma_{1}^{1 / 2}\right), \\
\operatorname{vec}\left(\Delta O^{(\text {ref })}\right) & =\mathcal{J}_{O^{\text {(ref })}, \mathcal{H}^{(1)} \operatorname{vec}\left(\Delta \mathcal{H}^{(1)}\right)}
\end{aligned}
$$

with

$$
\mathcal{J}_{O^{\text {(ref })}, \mathcal{H}^{(1)}}=\left(I_{n} \otimes\left(\frac{1}{2} S^{(1, \text { ref })} U_{1} \Sigma_{1}^{-1 / 2}\right)\right) S_{2}\left[\begin{array}{c}
\left(v_{1} \otimes u_{1}\right)^{T} \\
\vdots \\
\left(v_{n} \otimes u_{n}\right)^{T}
\end{array}\right]+\left(\Sigma_{1}^{1 / 2} \otimes S^{(1, \text { ref })}\left[\begin{array}{ll}
I_{n_{1}} & 0_{n_{1}, n_{2}}
\end{array}\right]\right)\left[\begin{array}{c}
B_{1}^{\dagger} C_{1} \\
\vdots \\
B_{n}^{\dagger} C_{n}
\end{array}\right],
$$

where $S_{2}$ is a selection matrix as in (B.3), $n_{1}=(p+1)\left(r^{(\mathrm{ref})}+r^{(1)}\right)$ and $n_{2}=q r^{(\mathrm{ref})}$ are the dimensions of $\mathcal{H}^{(1)}$ and $B_{i}$ and $C_{i}, i=1, \ldots, n$, are defined in Lemma 6 for $X=\mathcal{H}^{(1)}$. This proves the first part of Lemma 2.

For the second part of Lemma 2, choose any $j \in\left\{1, \ldots, N_{s}\right\}$ and let the dimensions of $\mathcal{H}^{(j, \text { ref })}$ and $\mathcal{H}^{(j, \text { mov })}$ be $n_{1} \times n_{3}$ and $n_{2} \times n_{3}$, respectively, where $n_{1}=(p+1) r^{\text {(ref) }}, n_{2}=(p+1) r^{(j)}$ and $n_{3}=q r^{\text {(ref) }}$. From (17) follows

$$
\Delta \bar{O}^{(j, \text { mov })}=\Delta\left(\mathcal{H}^{(j, \mathrm{mov})}\right) \mathcal{H}^{(j, \mathrm{ref}) \dagger} O^{(\mathrm{ref})}+\mathcal{H}^{(j, \mathrm{mov})} \Delta\left(\mathcal{H}^{(j, \mathrm{ref}) \dagger}\right) O^{(\mathrm{ref})}+\mathcal{H}^{(j, \mathrm{mov})} \mathcal{H}^{(j, \mathrm{ref}) \dagger} \Delta\left(O^{(\mathrm{ref})}\right)
$$

and in vectorized form

$$
\begin{aligned}
\operatorname{vec}\left(\Delta \bar{O}^{(j, \text { mov })}\right)=\left(\left(\mathcal{H}^{(j, \text { ref }) \dagger} O^{(\text {ref })}\right)^{T} \otimes I_{n_{2}}\right) \operatorname{vec}\left(\Delta \mathcal{H}^{(j, \text { mov })}\right)+\left(O^{(\text {ref }) T} \otimes \mathcal{H}^{(j, \text { mov })}\right) \operatorname{vec}\left(\Delta \mathcal{H}^{(j, \text { ref }) \dagger}\right) & +\left(\mathcal{H}^{(j, \text { mov })} \mathcal{H}^{(j, \text { ref }) \dagger} \otimes I_{n}\right) \operatorname{vec}\left(\Delta O^{(\text {ref })}\right) .
\end{aligned}
$$

The required perturbations in this equation are developed now, starting with $\Delta \mathcal{H}^{(j, r e f) \dagger}$. The pseudoinverse is defined with the SVD decomposition

$$
\mathcal{H}^{(j, \mathrm{ref})}=\left[\begin{array}{ll}
U_{1} & U_{0}
\end{array}\right]\left[\begin{array}{cc}
\Sigma_{1} & 0 \\
0 & \Sigma_{0}
\end{array}\right]\left[\begin{array}{l}
V_{1}^{T} \\
V_{0}^{T}
\end{array}\right]
$$

by $\mathcal{H}^{(j, \text { ref }) \dagger}=V_{1} \Sigma_{1}^{-1} U_{1}^{T}$, where $\Sigma_{1}$ is of size $n \times n$, as $\Sigma_{0}$ is asymptotically 0 . Then, a perturbation on $\mathcal{H}^{(j, \text { ref }) \dagger}$ writes as

$$
\Delta \mathcal{H}^{(j, \mathrm{ref}) \dagger}=\Delta\left(V_{1}\right) \Sigma_{1}^{-1} U_{1}^{T}-V_{1} \Sigma_{1}^{-1} \Delta\left(\Sigma_{1}\right) \Sigma_{1}^{-1} U_{1}^{T}+V_{1} \Sigma_{1}^{-1} \Delta\left(U_{1}^{T}\right) .
$$

Define $B_{i}$ and $C_{i}, i=1, \ldots, n$, in Lemma 6 for $X=\mathcal{H}^{(j, \text { ref })}$. Then, the vectorization of $\Delta U_{1}$ and $\Delta V_{1}$ is obtained with Lemma 6 as

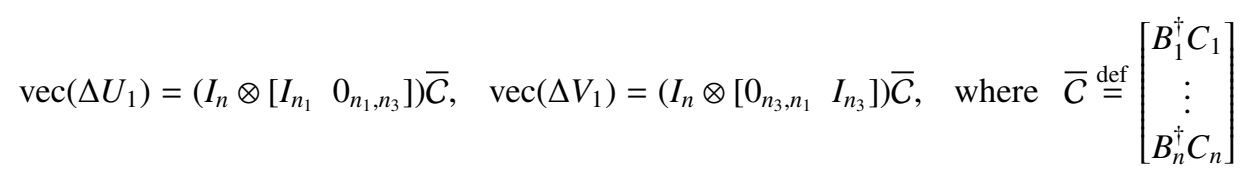


and with property (B.2) follows vec $\left(\Delta U_{1}^{T}\right)=\mathcal{P}_{n_{1}, n} \operatorname{vec}\left(\Delta U_{1}\right)$. The middle term in (C.5) writes as $V_{1} \Sigma_{1}^{-1} \Delta\left(\Sigma_{1}\right) \Sigma_{1}^{-1} U_{1}^{T}=$ $\sum_{i=1}^{n} \sigma_{i}^{-2} v_{i} \Delta \sigma_{i} u_{i}^{T}$. After vectorization and plugging in $\Delta \sigma_{i}$ from Lemma 6, it follows from (C.5)

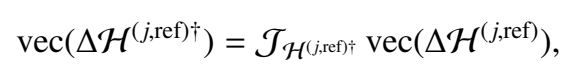

where

$$
\left.\left.\mathcal{J}_{\mathcal{H}^{(\text {j.ref })}=\left(U _ { 1 } \Sigma _ { 1 } ^ { - 1 } \otimes \left[0_{n_{3}, n_{1}}\right.\right.} I_{n_{3}}\right]\right) \bar{C}-\sum_{i=1}^{n} \sigma_{i}^{-2}\left(u_{i} v_{i}^{T} \otimes v_{i} u_{i}^{T}\right)+\left(I_{n_{1}} \otimes V_{1} \Sigma_{1}^{-1}\right) \mathcal{P}_{n_{1}, n}\left(I_{n} \otimes\left[\begin{array}{ll}
I_{n_{1}} & 0_{n_{1}, n_{3}}
\end{array}\right]\right) \bar{C}
$$

The perturbations $\Delta \mathcal{H}^{(j, \text { ref) }}$ in (C.6) and $\Delta \mathcal{H}^{(j, \text { mov })}$ in (C.4) are related to $\Delta \mathcal{H}^{(j)}$ by the selection matrices in (C.1) and it follows

$$
\operatorname{vec}\left(\Delta \mathcal{H}^{(j, \mathrm{ref})}\right)=\left(I_{n_{3}} \otimes S^{(j, \mathrm{ref})}\right) \operatorname{vec}\left(\Delta \mathcal{H}^{(j)}\right), \quad \operatorname{vec}\left(\Delta \mathcal{H}^{(j, \mathrm{mov})}\right)=\left(I_{n_{3}} \otimes S^{(j, \text { mov })}\right) \operatorname{vec}\left(\Delta \mathcal{H}^{(j)}\right)
$$

Then, the second part of Lemma 2 follows from (C.4) by plugging in (C.6) and (C.7) as well as (C.2), with the matrices

$$
\begin{aligned}
\mathcal{J}_{\bar{O}^{j}, O^{(\text {ref })}} & =\mathcal{H}^{(j, \text { mov })} \mathcal{H}^{(j, \text { ref }) \dagger} \otimes I_{n}, \\
\mathcal{J}_{\bar{O}^{j}, \mathcal{H}^{(j)}} & =\left(\left(\mathcal{H}^{(j, \text { ref }) \dagger} O^{(\text {ref })}\right)^{T} \otimes S^{(j, \text { mov })}\right)+\left(O^{(\text {ref }) T} \otimes \mathcal{H}^{(j, \text { mov })}\right) \mathcal{J}_{\mathcal{H}^{(j, \text { ref })}\left(I_{n_{3}} \otimes S^{(j, \text { ref })}\right) .}
\end{aligned}
$$

Proof of Lemma 3. From (19) follows $A=K^{-1} L$, where

$$
K \stackrel{\text { def }}{=} O^{(\mathrm{ref}) \uparrow T} O^{(\mathrm{ref}) \uparrow}+\sum_{j=1}^{N_{s}} \bar{O}^{(j, \mathrm{mov}) \uparrow T} \bar{O}^{(j, \mathrm{mov}) \uparrow}, \quad L \stackrel{\text { def }}{=} O^{(\mathrm{ref}) \uparrow T} O^{(\mathrm{ref}) \downarrow}+\sum_{j=1}^{N_{s}} \bar{O}^{(j, \mathrm{mov}) \uparrow T} \bar{O}^{(j, \mathrm{mov}) \downarrow} .
$$

Hence, $\Delta A=-K^{-1} \Delta K K^{-1} L+K^{-1} \Delta L=-K^{-1} \Delta K A+K^{-1} \Delta L$ and it follows

$$
\operatorname{vec}(\Delta A)=-\left(A^{T} \otimes K^{-1}\right) \operatorname{vec}(\Delta K)+\left(I_{n} \otimes K^{-1}\right) \operatorname{vec}(\Delta L) .
$$

Let $O \in \mathbb{R}^{(p+1) r \times n}$ be a placeholder for $O^{\text {(ref) }}$ or $\bar{O}^{(j, \text { mov })}$, where $r=r^{\text {(ref) }}$ or $r=r^{(j)}$, respectively. In order to obtain perturbations of the summands in (C.10), the perturbations $\Delta\left(O^{\uparrow T} O^{\uparrow}\right)$ and $\Delta\left(O^{\uparrow T} O^{\downarrow}\right)$ need to be evaluated.

Let $S_{\uparrow}^{(r)}$ and $S_{\downarrow}^{(r)}$ be the selection matrices in dependence of the number of sensors $r$ as defined in (B.4), such that $O^{\uparrow}=S_{\uparrow}^{(r)} O$ and $O^{\downarrow}=S_{\downarrow}^{(r)} O$. Then,

$$
\Delta\left(O^{\uparrow T} O^{\uparrow}\right)=\Delta\left(O^{T}\right) S_{\uparrow}^{(r) T} O^{\uparrow}+O^{\uparrow T} S_{\uparrow}^{(r)} \Delta(O) .
$$

and after vectorization and using property (B.2) it follows

$$
\operatorname{vec}\left(\Delta\left(O^{\uparrow T} O^{\uparrow}\right)\right)=\left(\left(O^{\uparrow T} S_{\uparrow}^{(r)} \otimes I_{n}\right) \mathcal{P}_{p r, n}+\left(I_{n} \otimes O^{\uparrow T} S_{\uparrow}^{(r)}\right)\right) \operatorname{vec}(\Delta O)
$$

Analogously, it holds

$$
\operatorname{vec}\left(\Delta\left(O^{\uparrow T} O^{\downarrow}\right)\right)=\left(\left(O^{\downarrow T} S_{\uparrow}^{(r)} \otimes I_{n}\right) \mathcal{P}_{p r, n}+\left(I_{n} \otimes O^{\uparrow T} S_{\downarrow}^{(r)}\right)\right) \operatorname{vec}(\Delta O) .
$$

Then, from (C.10) and (C.11) the assertion follows for $\Delta A$, where

$$
\begin{aligned}
& \mathcal{J}_{A, O^{\text {(ref })}}=-\left(A^{T} \otimes K^{-1}\right)\left(\left(O^{(\mathrm{ref}) \uparrow T} S_{\uparrow}^{\left(r^{(\mathrm{ref})}\right)} \otimes I_{n}\right) \mathcal{P}_{p r^{\text {(ref }, n}}+\left(I_{n} \otimes O^{(\mathrm{ref}) \uparrow T} S_{\uparrow}^{\left(r^{(\mathrm{reft})}\right)}\right)\right)
\end{aligned}
$$

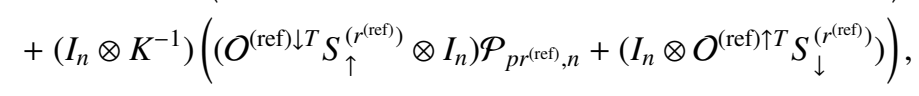

$$
\begin{aligned}
& \mathcal{J}_{A, \bar{O}^{j}}=-\left(A^{T} \otimes K^{-1}\right)\left(\left(\bar{O}^{(j, \mathrm{mov}) \uparrow T} S_{\uparrow}^{\left(r^{(j)}\right)} \otimes I_{n}\right) \mathcal{P}_{p r^{(j), n}}+\left(I_{n} \otimes \bar{O}^{(j, \mathrm{mov}) \uparrow T} S_{\uparrow}^{\left(r^{(j)}\right)}\right)\right) \\
& +\left(I_{n} \otimes K^{-1}\right)\left(\left(\bar{O}^{(j, \mathrm{mov}) \downarrow T} S_{\uparrow}^{\left(r^{(j)}\right)} \otimes I_{n}\right) \mathcal{P}_{p r^{(j), n}}+\left(I_{n} \otimes \bar{O}^{(j, \mathrm{mov}) \uparrow T} S_{\downarrow}^{\left(r^{(j)}\right)}\right)\right) .
\end{aligned}
$$


It remains the uncertainty of $C$. As $C$ is the first block row of (16), its perturbation can be written as

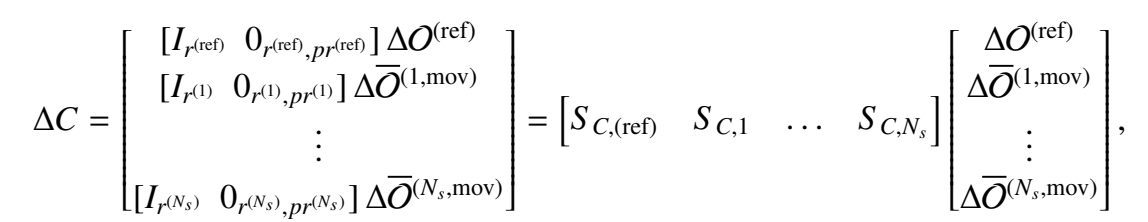

where

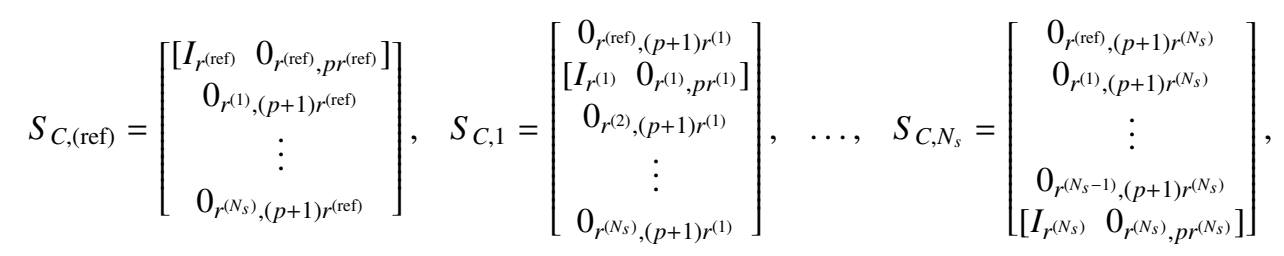

and it follows

$$
\Delta C=S_{C,(\mathrm{ref})} \Delta O^{(\mathrm{ref})}+\sum_{j=1}^{N_{s}} S_{C, j} \Delta \bar{O}^{(j, \mathrm{mov})}
$$

Vectorizing this equation leads to the assertion for $\Delta C$ with the matrices

$$
\mathcal{J}_{C, O^{(\text {ref })}}=I_{n} \otimes S_{C,(\mathrm{ref})}, \mathcal{J}_{C, \bar{O}^{j}}=I_{n} \otimes S_{C, j}
$$

Appendix C.2. Proofs of Section 6

Proof of Proposition 5. From the definition of $\widetilde{\varphi}_{i}$ follows

$$
\begin{aligned}
\Delta \widetilde{\varphi}_{i} & =\Delta\left(\mathrm{e}^{-\mathrm{i} \alpha_{i, k}}\right) C \phi_{i}+\mathrm{e}^{-\mathrm{i} \alpha_{i, k}} \Delta\left(C \phi_{i}\right) \\
& =-\mathrm{i} \mathrm{e}^{-\mathrm{i} \alpha_{i, k}} C \phi_{i} \Delta \alpha_{i, k}+\mathrm{e}^{-\mathrm{i} \alpha_{i, k}} \Delta\left(C \phi_{i}\right) .
\end{aligned}
$$

Consider the phase $\alpha_{i, k}$, which is obtained from the real and imaginary part of $s_{i, k}$ using the arctangent function and it follows

$$
\begin{aligned}
& \Delta \alpha_{i, k}=\Delta\left(\arctan \left(\mathfrak{J}\left(s_{i, k}\right) / \mathfrak{R}\left(s_{i, k}\right)\right)\right) \\
& =t_{i, k}^{-2}\left(-\mathfrak{J}\left(s_{i, k}\right) \Delta \mathfrak{R}\left(s_{i, k}\right)+\mathfrak{R}\left(s_{i, k}\right) \Delta \mathfrak{J}\left(s_{i, k}\right)\right) \\
& =t_{i, k}^{-2}\left(-\mathfrak{J}\left(s_{i, k}\right) e_{k}^{T} \Delta \mathfrak{R}\left(C \phi_{i}\right)+\mathfrak{R}\left(s_{i, k}\right) e_{k}^{T} \Delta \mathfrak{J}\left(C \phi_{i}\right)\right) \\
& =t_{i, k}^{-2}\left[-\mathfrak{J}\left(s_{i, k}\right) e_{k}^{T} \quad \mathfrak{R}\left(s_{i, k}\right) e_{k}^{T}\right]\left[\begin{array}{c}
\Delta \mathfrak{R}\left(C \phi_{i}\right) \\
\Delta \mathfrak{I}\left(C \phi_{i}\right)
\end{array}\right] .
\end{aligned}
$$

Plugging this result into the perturbation on $\widetilde{\varphi}_{i}$ yields

$$
\Delta \widetilde{\varphi}_{i}=\mathrm{e}^{-\mathrm{i} \alpha_{i, k}}\left(-\mathrm{i} t_{i, k}^{-2} C \phi_{i}\left[-\mathfrak{J}\left(s_{i, k}\right) e_{k}^{T} \quad \mathfrak{R}\left(s_{i, k}\right) e_{k}^{T}\right]+\left[\begin{array}{ll}
I_{r} & \mathrm{i} I_{r}
\end{array}\right]\right)\left[\begin{array}{l}
\Delta \mathfrak{R}\left(C \phi_{i}\right) \\
\Delta \mathfrak{J}\left(C \phi_{i}\right)
\end{array}\right] .
$$

From

$$
\begin{aligned}
\Delta\left(C \phi_{i}\right) & =\Delta C \phi_{i}+C \Delta \phi_{i} \\
& =\left(\phi_{i}^{T} \otimes I_{r}\right) \operatorname{vec}(\Delta C)+C \mathcal{J}_{\phi_{i}, A} \operatorname{vec}(\Delta A)
\end{aligned}
$$

where $\mathcal{J}_{\phi_{i}, A}$ is defined in Appendix B.3, follows

$$
\Delta \widetilde{\varphi}_{i}=\mathrm{e}^{-\mathrm{i} \alpha_{i, k}}\left(-\mathrm{i} t_{i, k}^{-2} C \phi_{i}\left[-\mathfrak{J}\left(s_{i, k}\right) e_{k}^{T} \quad \mathfrak{R}\left(s_{i, k}\right) e_{k}^{T}\right]+\left[\begin{array}{ll}
I_{r} & \mathrm{i} I_{r}
\end{array}\right]\right)\left[\begin{array}{ll}
C \mathfrak{R}\left(\mathcal{J}_{\phi_{i}, A}\right) & \mathfrak{R}\left(\phi_{i}\right)^{T} \otimes I_{r} \\
C \mathfrak{J}\left(\mathcal{J}_{\phi_{i}, A}\right) & \mathfrak{J}\left(\phi_{i}\right)^{T} \otimes I_{r}
\end{array}\right]\left[\begin{array}{c}
\operatorname{vec}(\Delta A) \\
\operatorname{vec}(\Delta C)
\end{array}\right],
$$

from where the assertion follows. 


\section{References}

[1] A. Benveniste, J.-J. Fuchs, Single sample modal identification of a nonstationary stochastic process, IEEE Transactions on Automatic Control AC-30 (1) (1985) 66-74.

[2] P. Van Overschee, B. De Moor, Subspace Identification for Linear Systems: Theory, Implementation, Applications, Kluwer, 1996.

[3] B. Peeters, G. De Roeck, Reference-based stochastic subspace identification for output-only modal analysis, Mechanical Systems and Signal Processing 13 (6) (1999) 855-878.

[4] A. Benveniste, L. Mevel, Nonstationary consistency of subspace methods, IEEE Transactions on Automatic Control AC-52 (6) (2007) 974984.

[5] H. Van der Auweraer, W. Leurs, P. Mas, L. Hermans, Modal parameter estimation from inconsistent data sets, in: Proc. 18th International Modal Analysis Conference, San Antonio, TX, USA, 2000, pp. 763-771.

[6] R. Mayes, S. Klenke, Consolidation of modal parameters from several extraction sets, Tech. rep., Sandia National Labs., Albuquerque, NM, and Livermore, CA (US) (2000).

[7] B. Cauberghe, P. Guillaume, B. Dierckx, P. Verboven, Identification of modal parameters from inconsistent data, in: Proc. 20th International Modal Analysis Conference, Los Angeles, CA, USA, 2002, pp. 809-815.

[8] E. Reynders, F. Magalhães, G. De Roeck, A. Cunha, Merging strategies for multi-setup operational modal analysis: application to the Luiz I steel arch bridge, in: Proc. 27th International Modal Analysis Conference, Orlando, FL, USA, 2009.

[9] J. Brownjohn, F. Magalhães, E. Caetano, A. Cunha, Ambient vibration re-testing and operational modal analysis of the Humber Bridge, Engineering Structures 32 (8) (2010) 2003-2018.

[10] F. Magalhães, E. Caetano, Á. Cunha, O. Flamand, G. Grillaud, Ambient and free vibration tests of the Millau Viaduct: Evaluation of alternative processing strategies, Engineering Structures 45 (2012) 372-384.

[11] L. Mevel, A. Benveniste, M. Basseville, M. Goursat, Blind subspace-based eigenstructure identification under nonstationary excitation using moving sensors, IEEE Transactions on Signal Processing SP-50 (1) (2002) 41-48.

[12] L. Mevel, M. Basseville, A. Benveniste, M. Goursat, Merging sensor data from multiple measurement setups for nonstationary subspace-based modal analysis, Journal of Sound and Vibration 249 (4) (2002) 719-741.

[13] M. Döhler, L. Mevel, Modular subspace-based system identification from multi-setup measurements, IEEE Transactions on Automatic Control. In press. doi:10.1109/TAC.2012.2193711

[14] M. Döhler, X.-B. Lam, L. Mevel, Crystal clear data fusion in subspace system identification and damage detection, in: Proc. 5th International Conference on Bridge Maintenance, Safety and Management, Philadelphia, PA, USA, 2010.

[15] M. Döhler, E. Reynders, F. Magalhães, L. Mevel, G. De Roeck, A. Cunha, Pre- and post-identification merging for multi-setup OMA with covariance-driven SSI, in: Proc. 28th International Modal Analysis Conference, Jacksonville, FL, USA, 2010.

[16] M. Döhler, P. Andersen, L. Mevel, Data merging for multi-setup operational modal analysis with data-driven SSI, in: Proc. 28th International Modal Analysis Conference, Jacksonville, FL, USA, 2010.

[17] M. Döhler, B. Jaishi, L. Mevel, J. Brownjohn, Data fusion for system identification of the Humber Bridge, in: Proc. 29th International Modal Analysis Conference, Jacksonville, FL, USA, 2011.

[18] A. Benveniste, B. Delyon, Using local tests to estimate convergence rates for identification, in: Proceedings of the 39th IEEE Conference on Decision and Control, 2000, pp. 1985-1990.

[19] A. Benveniste, M. Basseville, L. Mevel, Convergence rates for eigenstructure identification using subspace methods, in: Proceedings of the 39th IEEE Conference on Decision and Control, 2000, pp. 1550-1554.

[20] A. Chiuso, G. Picci, The asymptotic variance of subspace estimates, Journal of Econometrics 118 (1-2) (2004) $257-291$.

[21] T. Van den Boom, B. Haverkamp, Towards a state-space polytopic uncertainty description using subspace model identification techniques, International Journal of Control 76 (15) (2003) 1570-1583.

[22] R. Pintelon, P. Guillaume, J. Schoukens, Uncertainty calculation in (operational) modal analysis, Mechanical Systems and Signal Processing 21 (6) (2007) 2359-2373.

[23] E. Reynders, R. Pintelon, G. De Roeck, Uncertainty bounds on modal parameters obtained from stochastic subspace identification, Mechanical Systems and Signal Processing 22 (4) (2008) 948-969.

[24] M. Basseville, A. Benveniste, M. Goursat, L. Mevel, In-flight monitoring of aeronautic structures: vibration-based on-line automated identification versus detection, IEEE Control Systems Magazine 27 (5) (2007) 27-42.

[25] F. Magalhães, A. Cunha, E. Caetano, Vibration based structural health monitoring of an arch bridge: From automated OMA to damage detection, Mechanical Systems and Signal Processing 28 (2012) 212-228.

[26] E. Parloo, P. Guillaume, B. Cauberghe, Maximum likelihood identification of non-stationary operational data, Journal of Sound and Vibration 268 (5) (2003) 971-991.

[27] J. Brownjohn, Ambient vibration studies for system identification of tall buildings, Earthquake Engineering \& Structural Dynamics 32 (1) (2003) 71-95.

[28] D. Siringoringo, Y. Fujino, Noncontact operational modal analysis of structural members by laser doppler vibrometer, Computer-Aided Civil and Infrastructure Engineering 24 (4) (2009) 249-265.

[29] P. Mellinger, M. Döhler, L. Mevel, G. Broux, Data fusion for frequency domain stochastic subspace identification, in: Proceedings of the 5th European Conference on Structural Control (EACS), Genoa, Italy, 2012.

[30] J. Maeck, G. De Roeck, Description of Z24 benchmark, Mechanical Systems and Signal Processing 17 (1) (2003) $127-131$.

[31] D. Miller, R. de Callafon, Efficient identification of input dynamics for correlation function-based subspace identification, in: Proc. 18th IFAC World Congress, Milan, Italy, 2011, pp. 6511-6516.

[32] B. Peeters, G. De Roeck, Stochastic system identification for operational modal analysis: a review, Journal of Dynamic Systems, Measurement, and Control 123 (4) (2001) 659-667.

[33] B. Peeters, C. Ventura, Comparative study of modal analysis techniques for bridge dynamic characteristics, Mechanical Systems and Signal Processing 17 (5) (2003) 965-988. 
[34] W. Gersch, On the achievable accuracy of structural system parameter estimates, Journal of Sound and Vibration 34 (1) (1974) 63-79.

[35] M. Döhler, L. Mevel, Robust subspace based fault detection, in: Proc. 18th IFAC World Congress, Milan, Italy, 2011.

[36] E. Reynders, Uncertainty quantification in operational modal analysis: Application to a damaged pre-stressed concrete bridge, in: Proc. 4th Experimental Vibration Analysis for Civil Engineering Structures (EVACES), Varenna, Italy, 2011.

[37] G. Golub, C. Van Loan, Matrix computations, 3rd Edition, Johns Hopkins University Press, 1996. 